\title{
Performance of ANAIS-112 experiment after the first year of data taking
}

\author{
J. Amaré1,3, S. Cebrián ${ }^{1,3}$, I. Coarasa ${ }^{1,3}$, C. Cuesta ${ }^{1,4}$, E. García ${ }^{1,3}$, M. Martínez ${ }^{2,3, a}{ }_{\mathbb{D}}$, M. A. Oliván ${ }^{1,5}$,

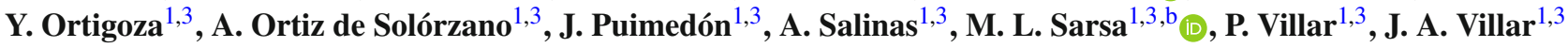 \\ ${ }^{1}$ Laboratorio de Física Nuclear y Astropartículas, Universidad de Zaragoza, C/ Pedro Cerbuna 12, 50009 Zaragoza, Spain \\ ${ }^{2}$ Fundación Agencia Aragonesa para la Investigación y el Desarrollo (ARAID), Gobierno de Aragón, Avenida de Ranillas 1-D, 50018 Zaragoza, \\ Spain \\ ${ }^{3}$ Laboratorio Subterráneo de Canfranc, Paseo de los Ayerbe s.n., 22880 Canfranc Estación, Huesca, Spain \\ ${ }^{4}$ Present Address: Centro de Investigaciones Energéticas, Medioambientales y Tecnológicas, CIEMAT, 28040 Madrid, Spain \\ ${ }^{5}$ Present Address: Fundación CIRCE, 50018 Zaragoza, Spain
}

Received: 5 December 2018 / Accepted: 18 February 2019 / Published online: 12 March 2019

(C) The Author(s) 2019

\begin{abstract}
ANAIS is a direct detection dark matter experiment aiming at the study of the annual modulation expected in the interaction rate. It uses same target and technique as the DAMA/LIBRA experiment, which reported a highly significant positive modulation compatible with that expected for dark matter particles distributed in the galactic halo. However, other very sensitive experiments do not find any hint of particles with the required properties, although comparison is model dependent. In 2017, ANAIS-112 experiment was installed at the Canfranc Underground Laboratory (LSC), in Spain, and after the commissioning run for calibration and general assessment, ANAIS-112 started data taking in dark matter mode on August 3rd, 2017. It consists of nine $\mathrm{NaI}(\mathrm{Tl})$ modules, amounting $112.5 \mathrm{~kg}$ of mass in total. Here we report on the experimental apparatus and detector performance after the first year of data taking. Total live time available amounts to 341.72 days, being the corresponding exposure $105.32 \mathrm{~kg} \times$ year. ANAIS-112 has achieved an analysis energy threshold of 1 keVee and an average background in the region of interest, from 1 to $6 \mathrm{keVee}$, of 3.6 counts $/ \mathrm{keVee} / \mathrm{kg} /$ day after correcting by the event selection efficiencies. In these conditions, ANAIS-112 will be able to test the DAMA/LIBRA result at three sigma level in five years of data taking.
\end{abstract}

J. A. Villar: Deceased.

\footnotetext{
a e-mail: mariam@unizar.es
}

be-mail: mlsarsa@unizar.es

\section{Introduction}

There is overwhelming evidence accumulating over the last decades supporting that a large fraction of the Universe energy-mass budget is not explained in the frame of the standard model of the particle physics if assuming the cosmological standard model [1]. The solution to this puzzle should be searched for in any possible direction: looking for extensions of the particle physics standard model while working on new theoretical frameworks for gravity and/or dynamics. The complex nature of the dark matter (DM) and dark energy (DE) problems makes them one the main challenges for present particle physics and cosmology. DM can be understood as a new non-zero-mass particle having a very low interaction probability with baryonic matter. Axions and Weakly Interacting Massive Particles (WIMPs) are among the preferred candidates [2].

Despite the wide experimental effort devoted to the understanding of the particle DM nature, no unambiguous claim has been reported by direct [3], indirect [4] or accelerator searches [5] so far. However, there is a long-standing positive result on the field: the observation of a high statistically significant annual modulation in the detection rate, compatible with that expected for galactic halo dark matter particles, at the DAMA/LIBRA experiment [6-8], in operation at the Gran Sasso National Laboratory, in Italy. This result has neither been reproduced by any other experiment, nor ruled out in a model independent way, although compatibility in most conventional WIMP-DM scenarios is actually disclaimed [914].

ANAIS stands for Annual modulation with NaI Scintillators, and has as main goal the testing of DAMA/LIBRA result 
using the same target and technique. ANAIS-112 consists of $112.5 \mathrm{~kg}$ of $\mathrm{NaI}(\mathrm{Tl})$ detectors, and it was installed in 2017 at the Canfranc Underground Laboratory, LSC, in Spain. The dark matter run started on the 3rd of August, 2017, being now completed the first year of data taking. ANAIS-112 can test the DAMA/LIBRA result in a model independent way at 3 sigma C.L. in 5 years of data taking, having a significant discovery potential if the modulation signal is effectively due to dark matter particles [15]. A different experiment, having different residual cosmic ray flux and different environmental conditions than DAMA/LIBRA has, could bring a new insight in the DAMA/LIBRA observation: confirmation of a modulation having the same phase and amplitude would be very difficult to explain as effect of backgrounds or systematics. In this sense, it is worth remarking here the main differences among ANAIS-112 and DAMA/LIBRA operation conditions. First, the LSC rock overburden is lower than that of Gran Sasso (800 m versus $1400 \mathrm{~m}$ rock), allowing for a larger expected contribution from cosmogenic related backgrounds in ANAIS-112 than in DAMA/LIBRA. On the other hand, ANAIS-112 experimental layout disposes of an active muon veto system that allows tagging and removing muon related events and monitoring the onsite muon flux. Second, the contribution from radiogenic related backgrounds is mainly independent on the laboratory overburden. Radon contribution is dependent on the local geological conditions (for production) and experimental spatial conditions, for instance ventilation of the spaces, while cosmogenically activated isotopes contribution is dependent on the exposure to cosmic rays and time spent underground. Third, PMT noise events would be monitored along the second year of operation with a blank module specifically commissioned for that goal, which is taking data since August 2018 at LSC.

Other efforts sharing ANAIS goal in the international dark matter community are the COSINE-100 experiment, taking data also in dark matter mode at Yang-Yang Underground Laboratory, South Korea [16,17], and in longer-term the SABRE project, aiming at installing twin detectors in Australia and Italy [18] and the COSINUS project, aiming at the R\&D on cryogenic detectors based on $\mathrm{NaI}$ [19].

In this paper, we describe the most relevant experimental parameters of ANAIS-112 set-up and present the experiment performance along the first year of data taking. The structure of the paper is as follows: Sect. 2 describes the ANAIS-112 experimental layout, including electronics and data analysis; Sect. 3 focuses on the energy calibration of the experiment, both at low and high energy; Sect. 4 describes the procedures aiming at selecting sodium iodide bulk scintillation events; Sect. 5 summarizes the total efficiencies estimated for the detection and selection of events in the Region of Interest (ROI) and Sect. 6 briefly describes the ANAIS112 background sources, referencing conveniently to other papers where this issue is more thoroughly studied. As far as stability of the environmental conditions is important for the implications of the annual modulation analysis intended, Sect. 7 describes the temporal variability of relevant experimental parameters along the first year of data taking. Finally, a brief summary and outlook are presented in Sect. 8 .

\section{The ANAIS-112 experiment}

ANAIS-112 experiment uses nine $\mathrm{NaI}(\mathrm{Tl})$ modules produced by Alpha Spectra Inc. (AS) in Colorado (US). These modules have been manufactured along several years and then shipped to Spain, avoiding air travel in order to prevent from cosmogenic activation, arriving at LSC the first of them at the end of 2012 and the last one by March, 2017. Each crystal is cylindrical (4.75" diameter and 11.75 " length), with a mass of $12.5 \mathrm{~kg}$. NaI(Tl) crystals were grown from selected ultrapure $\mathrm{NaI}$ powder and housed in OFE (Oxygen Free Electronic) copper; the encapsulation has a Mylar window allowing low energy calibration using external gamma sources. Two highly efficient Hamamatsu R12669SEL2 photomultipliers (PMTs) were coupled through quartz windows to each crystal at LSC clean room. In the following we will refer as PMT-0 (PMT-1) to the units placed on the East (West) side of the shielding. All PMT units, as well as many of the materials used in the building of the detectors, have been screened for radiopurity using germanium detectors at the LSC low background facilities and their contribution to the experiment background has been estimated [20,21]. The ANAIS-112 shielding consists of $10 \mathrm{~cm}$ of archaeological lead, $20 \mathrm{~cm}$ of low activity lead, an anti-radon box (continuously flushed with radon-free nitrogen gas), an active muon veto system made up of 16 plastic scintillators designed to cover top and sides of the whole ANAIS set-up (see Fig. 1) and $40 \mathrm{~cm}$ of neutron moderator (a combination of water tanks and polyethylene blocks). The hut housing the experiment is located at the hall B of LSC under 2450 m.w.e. rock overburden.

\subsection{ANAIS-112 DAQ system}

The ANAIS-112 electronic chain and data acquisition system (DAQ) are designed to assure the lowest trigger level in stable conditions over a long period of time. In Fig. 2 we show a sketch of the main components of the electronic chain for a single module (upper panel) and for the total system (lower panel). A more detailed description of the DAQ system can be found in [22].

The first amplification stage for each PMT signal is carried out by custom-made pre-amplifiers located inside the ANAIS hut, just outside the anti-radon box, in order to minimize the length of the cables and then, optimize the signal/noise ratio. The rest of the ANAIS-112 electronics (mainly based on VME modules) are located outside the hut, in an air- 

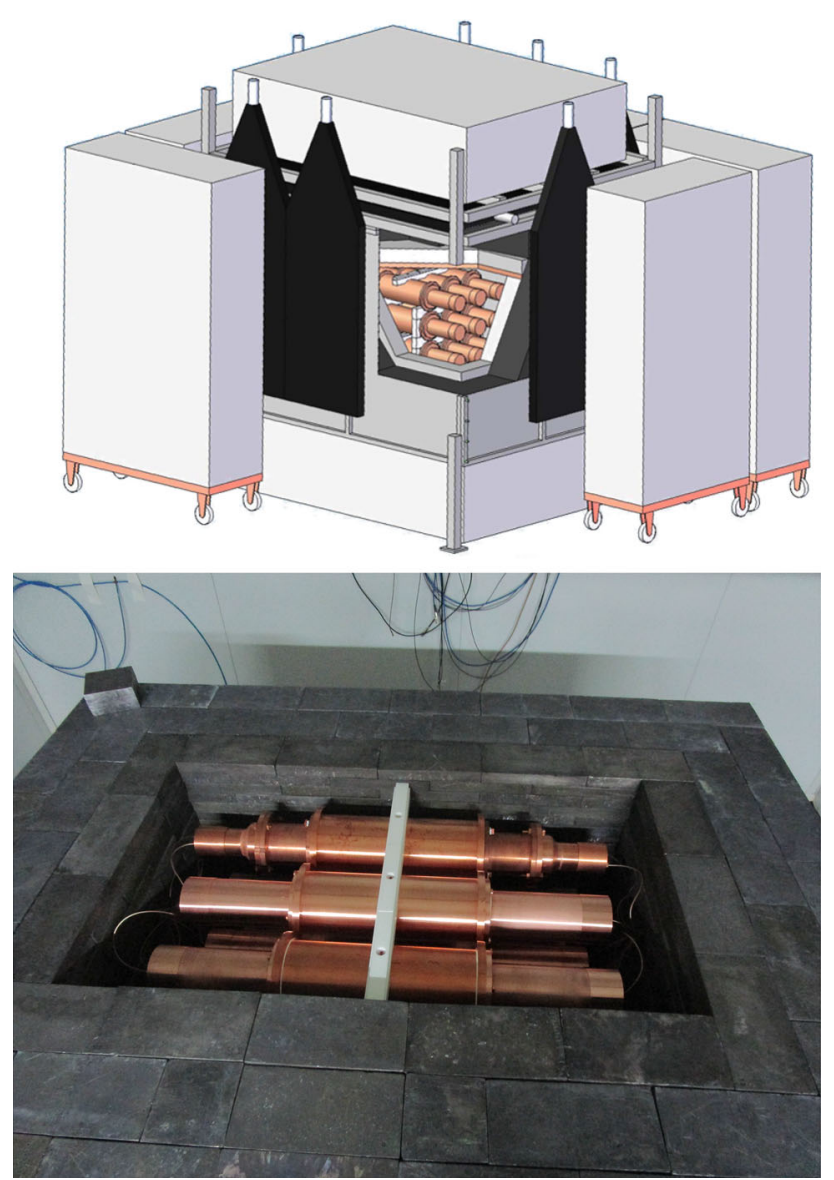

Fig. 1 Upper panel: schematic view of the ANAIS-112 full set-up; it consists of nine NaI modules (each crystal coupled to 2 PMT units) placed in a $3 \times 3$ array, a lead shielding $(10 \mathrm{~cm}$ archaeological lead + $20 \mathrm{~cm}$ low activity lead), an anti-radon box continuously flushed with radon-free nitrogen gas, an active veto system (made of 16 plastic scintillators), and $40 \mathrm{~cm}$ of neutron shielding (water tanks and polyethylene blocks). Lower panel: picture taken while ANAIS-112 commissioning; detail on the calibration system for positioning the $\mathrm{Cd}$ sources in front of the Mylar window of each module can be also observed

conditioned rack cabinet whose temperature is controlled and continuously monitored. The PMT's high voltage (HV) supply is provided by a CAEN SY2527 Universal Multichannel Power Supply, which allows continuous monitoring of the individual voltages.

The charge signal coming from every PMT is separately processed and recorded. Each one is divided into: (1) a trigger signal; (2) a so-called low energy (LE) signal that goes to the digitizer; and (3) a high energy (HE) signal, conveniently attenuated for high energy events. The trigger of each PMT signal is performed by a CAEN N843 constant fraction discriminator (CFD), with a threshold level low enough to effectively trigger at the photoelectron (phe) level, while the single module trigger is done by the coincidence (logical AND) of the two PMT triggers in a $200 \mathrm{~ns}$ window. The HE signals are fed into CAEN V792 QDC (charge-to-digital-
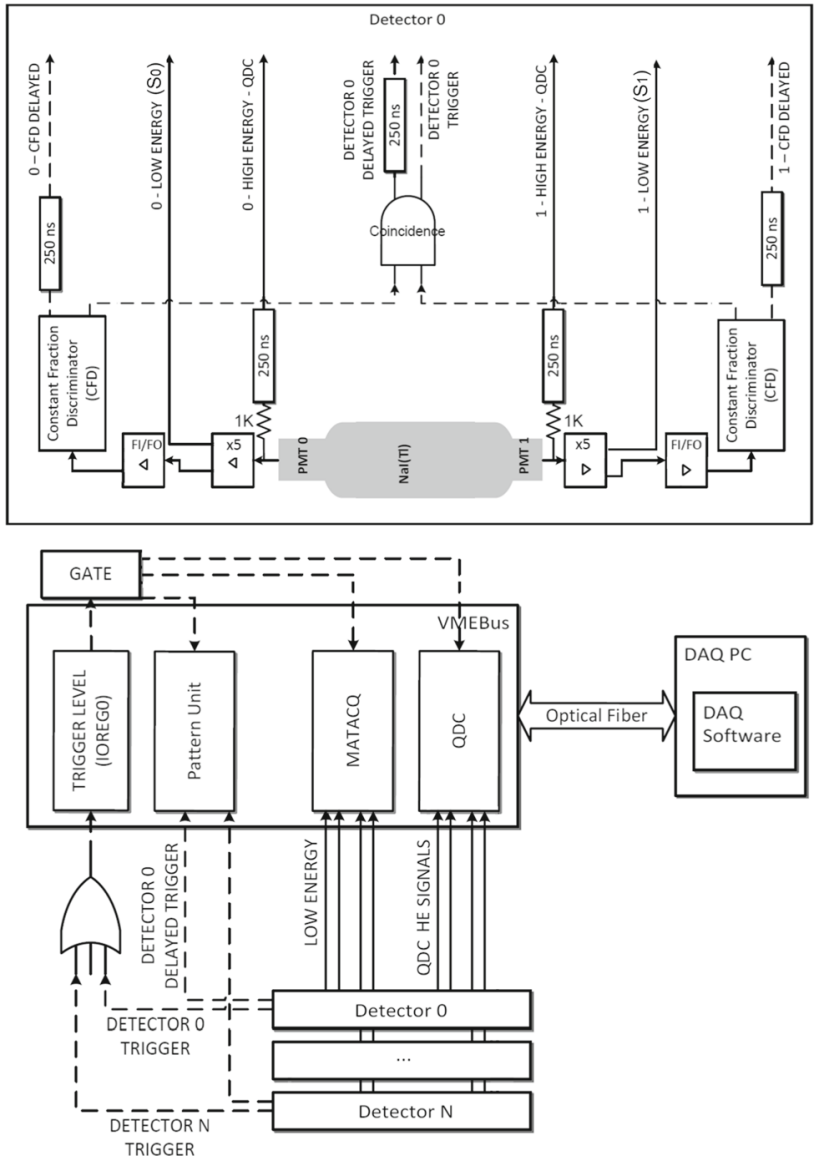

Fig. 2 Electronics front-end of the ANAIS-112 DAQ acquisition system for one single module (upper panel) and the complete system (lower panel)

converter) modules, with $1 \mu \mathrm{s}$ integration window and 12 bits resolution. The LE signals ( $\mathrm{S}_{0}$ and $\mathrm{S}_{1}$ in Fig. 2 ) are sent to MATACQ-CAEN V1729A digitizers, which continuously sample the waveforms at $2 \mathrm{Gs} / \mathrm{s}$ with high resolution (14 bits) and store them in circular buffers.

The main acquisition trigger, that is the logical OR of the nine modules individual trigger, is sent to a CAEN V977 I/O Register (IOREG0), whose state ON represents the DAQ busy state and is used for measuring the dead time, until it is reset via a VME command by the DAQ program. It fires the QDC conversion and the transfer of 2520 points-length waveforms (including 500 pre-trigger points) from the MATACQ buffers to the DAQ computer memory. The I/O Register also opens $1 \mu$ s window via a gate generator (GATE) which defines the coincidence among modules. In addition, a Pattern Unit module registers which detectors have triggered and allows the DAQ system to acquire only these data, reducing the dead time and saving disk space.

The building of the spectra is done by software (off-line) by integrating the $S_{0}(t)$ and $S_{1}(t)$ waveforms and adding them for each module: this variable will be called in the 


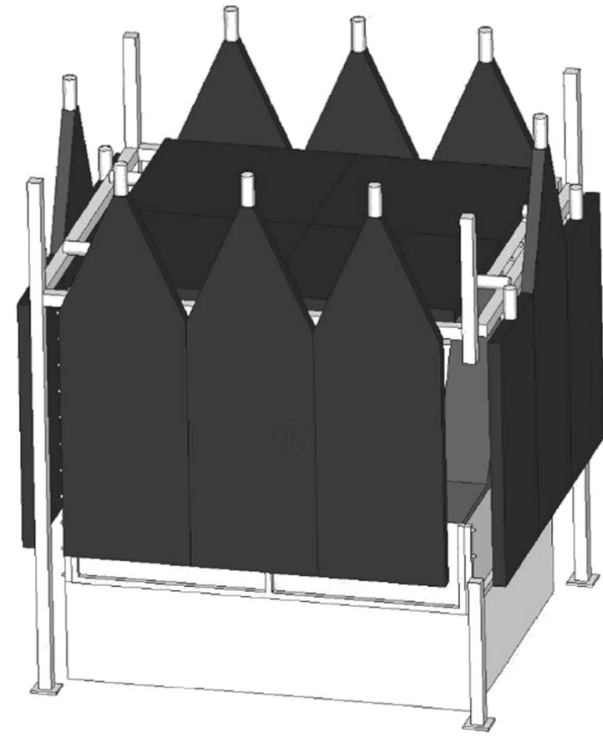

Fig. 3 Muon Veto System layout in ANAIS-112 setup. It consists of 16 plastic scintillators covering top and lateral sides of the shielding (see Fig. 1). It allows tagging muon related events in the NaI crystals by off-line analysis. Trigger rate of the veto system is $5.6 \mathrm{~Hz}$ before applying PSA techniques to select muon events from plastic scintillators background; the rate of muons finally identified is below $40 \mathrm{mHz}$

following pulse area. These waveforms also serve to perform Pulse Shape Analysis (PSA) in order to select bulk scintillation events in the NaI crystals from PMT-origin events (see Sect. 4) and to distinguish alpha interactions from beta/gamma ones (Sect. 3.1).

The real time master clock is generated by the VME/PCI bridge, started at the beginning of the run, and it is stored in a 64 bit scaler. Another VME/PCI bridge controller clock is used to measure the live time. This clock (also stored in a 64 bit scaler) is stopped when the IOREG enters in busy state and it is started when the state is reset. The dead time is dominated by the waveform digitization and transfer from the MATACQ board and amounts to about $5 \mathrm{~ms}$ per event. For the first year ANAIS-112 average trigger rate, about $5.5 \mathrm{~Hz}$ (see Sect. 2.4), this implies a $2.8 \%$ dead time.

\subsection{ANAIS-112 muon veto system}

The role played by the veto scintillator system in ANAIS-112 data selection and annual modulation analysis is very important. It is well known that the underground muon flux has an annual modulation [23-26] which could cause muon related events in dark matter detectors to mimic the dark matter modulation. However, different phases from DAMA/LIBRA and underground muon flux modulations have been determined and DAMA/LIBRA collaboration completely discard muons as possible explanation of their signal [27]. This is because direct effect of muon interactions in the $\mathrm{NaI}(\mathrm{Tl})$ detectors should contribute outside the ROI for the dark matter modulation analysis, at very high energies, for instance, and if present in DAMA/LIBRA ROI, they should also contribute both, to events in coincidence among several detectors and in other energy regions, which has not been observed by DAMA/LIBRA collaboration. However, contribution from other muon related events could fall into the ROI and correspond to single hit events: muons can produce secondary neutrons in the setup materials, either prompt or delayed neutrons in a wide energy range; muons can interact in the PMT, quartz optical windows, and other detectors components producing there weak scintillation signals [28]; and last, because of the very slow scintillation observed in $\mathrm{NaI}(\mathrm{Tl})$ [29], the tail of the scintillation pulse produced by a large energy deposition from a muon in one of the crystals could trigger several times the ANAIS-112 DAQ system (as explained in Sect. 4.1). The ANAIS-112 veto scintillator system allows to correlate muon events in the plastic scintillators with energy depositions in the $\mathrm{NaI}(\mathrm{Tl})$ crystal in order to better understand and monitor these effects.

ANAIS-112 muon veto system consists of 16 plastic scintillators having different shapes which cover all but the bottom of the ANAIS-112 lead shielding (see Fig. 3). A tagging strategy instead of a hardware vetoing strategy has been followed in the design of this system. This is because the goal of the muon veto system is to detect the residual muon flux at the detectors position in order to discard coincident events in the $\mathrm{NaI}(\mathrm{Tl})$ crystals, but also to study possible correlations between muon hits in the plastic scintillators and events in the $\mathrm{NaI}(\mathrm{Tl})$ crystals, specially in the ROI. In order to minimize losing muon events below plastic scintillator trigger threshold, the resulting trigger rate on the veto system was high, $5.6 \mathrm{~Hz}$, being dominated by background events from the plastic scintillators. Because the plastic scintillators used in the veto system have a strong particle discrimination capability, background events are much faster than muon events, and they can be removed by Pulse Shape Analysis techniques, applied off-line. The muon detection front-end electronics were designed to provide enough information to allow this off-line particle discrimination: for each event the whole pulse area and tail pulse area are saved, being muon events discriminated by the ratio of these two parameters quite efficiently [22]. Muon selection parameters and operation conditions of the plastic scintillators have been chosen to minimize the rejection of real muon events. Some other relevant information is also saved for every event: multiplicity trigger pattern and real time tagging. The DAQ software of the veto system is almost the same developed for the $\mathrm{NaI}(\mathrm{Tl})$ detectors DAQ, but it is independently run in a different computer and with a different configuration. It is launched remotely by the ANAIS-112 DAQ scripts to guarantee same start counting in clock scalers, having been the time correlation between both 
Table 1 Average rate of muons (after PSA) in the five sides of ANAIS112 Veto System and corresponding standard deviation of the population

\begin{tabular}{lll}
\hline Side & Average Muon rate $\mu \mathrm{s}^{-1}$ & Standard deviation \\
\hline Top & 0.01167 & 0.00055 \\
North & 0.00766 & 0.00037 \\
South & 0.00787 & 0.00041 \\
East & 0.00598 & 0.00048 \\
West & 0.00598 & 0.00035 \\
\hline
\end{tabular}

Table 2 Average rate of muons (after PSA) in coincidence among the different sides of ANAIS-112 Veto System and standard deviation of the population

\begin{tabular}{lll}
\hline Side-side coincidence & Average Muon rate $\mu \mathrm{s}^{-1}$ & Standard deviation \\
\hline Top-North & 0.0037 & 0.0022 \\
Top-South & 0.0014 & 0.0008 \\
Top-East & 0.0009 & 0.0006 \\
Top-West & 0.0011 & 0.0006 \\
North-South & 0.0004 & 0.0002 \\
East-West & 0.00008 & 0.00005 \\
\hline
\end{tabular}

DAQ systems thoroughly studied while commissioning and testing, both at surface and underground LSC facilities [22].

The residual muon flux at the LSC has been recently determined to be $4.4 \times 10^{-3} \mu \mathrm{s}^{-1} \mathrm{~m}^{-2}$ [30], which is over four orders of magnitude lower than the surface muon flux, but enough to have some influence in the ANAIS detectors. This flux is higher than that measured at Gran Sasso Laboratory, where the DAMA/LIBRA experiment is in operation by about one order of magnitude [23]. The larger muon flux at LSC would produce a different (probably higher) modulation amplitude in ANAIS-112 data if muon interactions are somehow behind DAMA/LIBRA result and contribute to the ROI in an unsuspected way.

Average muon rates observed along the first year of operation of ANAIS-112 are shown in Table 1 for each side of the experiment layout. Table 2 shows the average rate of coincidences in veto scintillators from two different sides. The latter is more cleary dependent on the shape of the rock overburden profile over the LSC, which is quite asymmetric, being lower on the North side. However, the angular dependence of the muon flux at LSC Hall B is not well known and then, muon flux estimation is difficult. Using only the top side information, without any correction, we get an average muon flux value of $5.6 \times 10^{-3} \mu \mathrm{s}^{-1} \mathrm{~m}^{-2}$.

\subsection{ANAIS-112 data-taking}

ANAIS-112 detector was assembled in early 2017 and the commissioning phase was performed from March to July.

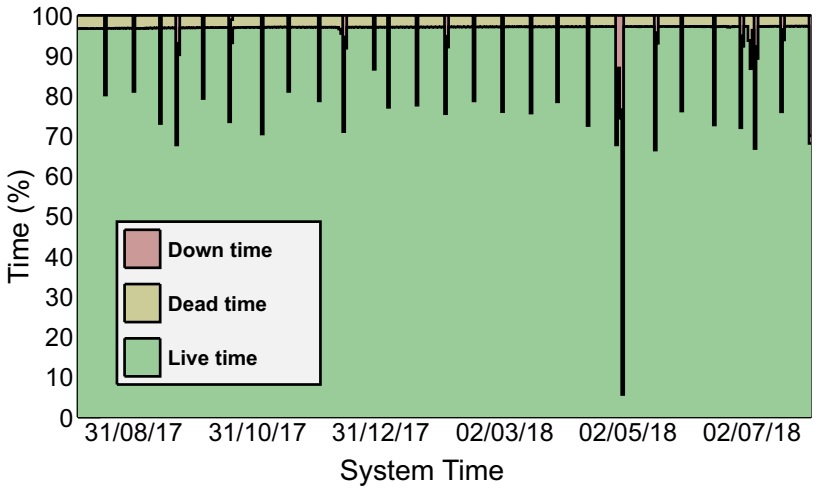

Fig. 4 Distribution of live, dead and down time during the first year of ANAIS-112 DM data taking. Total numbers for this first year are: $94.5 \%$ live time, $2.9 \%$ dead time, and $2.6 \%$ down time. Down time periods correspond mostly to periodical calibration with low energy external sources, but some incidences are clearly observed: an electrical power failure at the LSC (end of April-beginning of May 2018) and a failure on ANAIS DAQ computer disk at the beginning of July 2018. Colors referenced are available in the online version of the paper

The dark matter data taking started on the 3rd of August 2017. Dark matter runs last for about two weeks and in between we perform a ${ }^{109} \mathrm{Cd}$ calibration run, that usually takes about 3-4 h.

One of the most important assets of ANAIS-112 in the search for the annual modulation is the very high duty cycle achieved. Figure 4 shows the fraction of live, dead and down time corresponding to the first year of the ANAIS-112 dark matter run. Total numbers are the following: $94.5 \%$ live time, $2.9 \%$ dead time, and $2.6 \%$ down time. Excluding the down time, which is mostly due to periodical calibration of the modules using external gamma sources (seen as periodical spikes in Fig. 4), we get a live time of $97 \%$ in DM run mode. These numbers are expected to improve for the second year of data taking, provided some small incidences affecting electrical supply of the LSC and maintenance works can be avoided. Some of those happening in this period can be clearly observed in Fig. 4: an electrical power failure at LSC (end April - beginning May 2018) and a failure on ANAIS DAQ computer disk (beginning of July 2018). From the 3rd of August 2017 to the 31st of July 2018, 341.72 days (live time) are available for the annual modulation analysis.

Using this live time distribution (day by day) along the first year of data taking we can calculate $\alpha$, the average of $\cos ^{2}(\omega t)$, obtaining a value of 0.46850 , and $\beta=0.00466$, the average of $\cos (\omega t)$, assuming $\omega=2 \pi / 365$ days $^{-1}$. These parameters should be 0.5 and 0.0 , respectively, for a perfect coverage of the whole year-period.

\subsection{ANAIS-112 modules performance}

Total trigger rate evolution along the first year of data (shown in black solid dots in Fig. 5) shows a slow but clear decrease. 


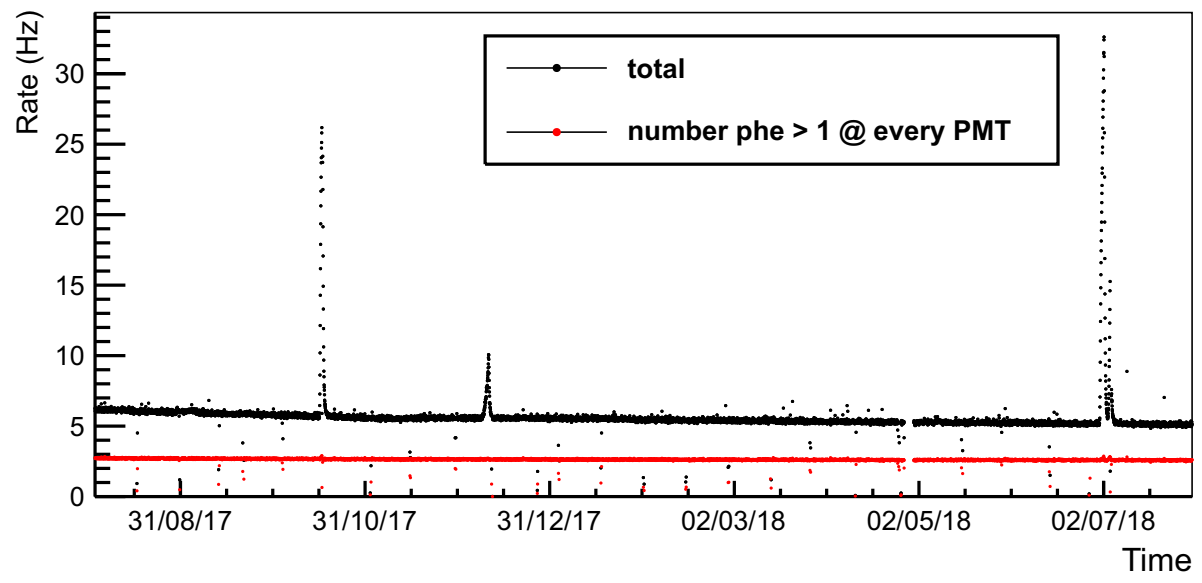

Fig. 5 Trigger rate (calculated in $3600 \mathrm{~s}$ time bins) during the first year of ANAIS-112 DM data taking: total trigger rate is shown in black, trigger rate of events having more than one phe in the pulse at each PMT is shown in red. Strong increases in the total trigger rate of the experiment are related with exhausting the liquid nitrogen dewar providing the radon free gas flux continuously coming into the shielding, but the increase is not observed in events having more than one phe in each PMT signal (red solid dots), which points to PMT related events still to be understood (see text for more information). Colors referenced are available in the online version of the paper
The liquid nitrogen supply was exhausted three times in this period, and the total trigger rate of the experiment has shown to be very sensitive to the complete exhaustion of nitrogen gas flux entering into the ANAIS-112 shielding (see Fig. 37). However, the rate increase in these periods is not directly explained by background events originating from gammas emitted by the Rn daughters: in Fig. 5 it is shown as red solid dots the event rate after rejecting those events having only one phe in each PMT, which has been very stable along the whole ANAIS-112 first year data. We can conclude, most of the events triggering in the periods without nitrogen supply only have one phe in each PMT, which, by the way, usually dominate also the total trigger rate in ANAIS-112. These events are considered to be PMT-related events, but we are still working to understand their rate correlation with the nitrogen gas flux coming into the shielding using a blank module, specifically commissioned for the second year of data taking with this goal. These events are very efficiently removed by our event selection procedure (see Sect. 4) and they do not compromise the ANAIS-112 scientific goals. Moreover, in collaboration with LSC staff, radon-free gas supply has been improved for the second year of ANAIS-112 operation.

We can also look at the evolution in time of the coincident events rate, for instance those having multiplicity 2 (two detectors with a signal within the coincidence window) are shown in red in Fig. 6. These events should track the contribution from different background sources, as radon intrusion into the shielding or external gamma flux. Figure 6 shows the rate of events in coincidence with $609 \mathrm{keV}, 1120 \mathrm{keV}$ or $1764 \mathrm{keV}$ gamma lines as black solid dots, which are all attributable to ${ }^{222} \mathrm{Rn}$ progeny. These rates are also very stable along the year of data taking.
Regarding the response of the detectors, we compute the light collection by comparing the charge collected at the PMTs following a known energy deposition in the crystal (in our case, the $22.6 \mathrm{keV}$ line of a ${ }^{109} \mathrm{Cd}$ source) with the charge distribution of a single phe, the so-called Single Electron Response (SER). In order to obtain a population of phe not biased in amplitude by the trigger threshold, we select very low-energy background events, for which phe can be individually counted, and take the last one in the waveform, integrating the charge in a narrow window around the phe maximum: [ $-30 \mathrm{~ns},+60 \mathrm{~ns}$ ]. In order to do so we use a peak identification algorithm [22], based on a low pass filter and the detection of a sign change in the derivative waveform. It uses a very low software threshold in order to rescontruct the SER down to the baseline noise. This routine performs very well at very low energy, being its efficiency limited by the phe overlap due to their non-zero width. Some examples of pulses with a low number of phe can be found in Sect. 4.2, Fig. 19.

The light collected at every PMT is then calculated as the ratio between the mean pulse area corresponding to the $22.6 \mathrm{keV}$ calibration line and the mean of the SER pulse area distribution [31]. Figure 7 shows the distribution of the pulse area for the 18 PMTs used in ANAIS-112 set-up; this distribution is determined using background data grouped in two weeks periods, and the figure shows the distribution corresponding to one of them. It must be remarked the outstanding light collection measured for the nine AS modules, at the level of 15 phe/keV (see Table 3). Only D6 module shows a light collection slightly below 13 phe/keV. This high light output has a direct impact in both resolution and energy threshold, but it also allows to improve strongly the signal vs noise 
Fig. 6 Trigger rate (calculated in $3600 \mathrm{~s}$ time bins) during the first year of ANAIS-112 DM data taking: double coincidences rate (multiplicity 2) is shown in black, rate of events in coincidence with $609 \mathrm{keV}$, $1120 \mathrm{keV}$ or $1764 \mathrm{keV}$, is shown in red. Colors referenced are available in the online version of the paper

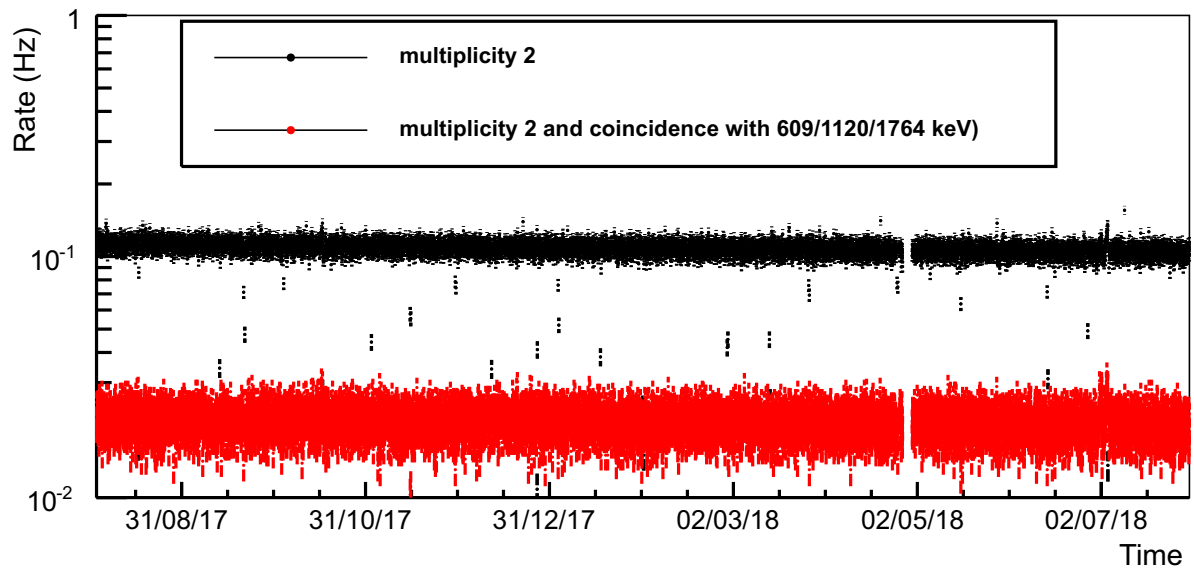

Fig. 7 Example of SER area distributions for the 2 PMT $\times 9$ detectors of ANAIS-112. They are obtained for background runs every two weeks, selecting the last peak in pulses having a very low number of phe, in order to not be biased by the trigger threshold (see text for more information). At each panel, the first digit represents the detector number and the second one the PMT (PMT-0,PMT-1). The response to individual photons of a PMT unit is characteristic and strongly dependent on the HV supply applied. The units 41 and 51 (corresponding to D4 and D5 modules) were operated at higher $\mathrm{HV}$, and this can be observed as a broader SER area distribution and a higher mean value, as the PMT gain is higher

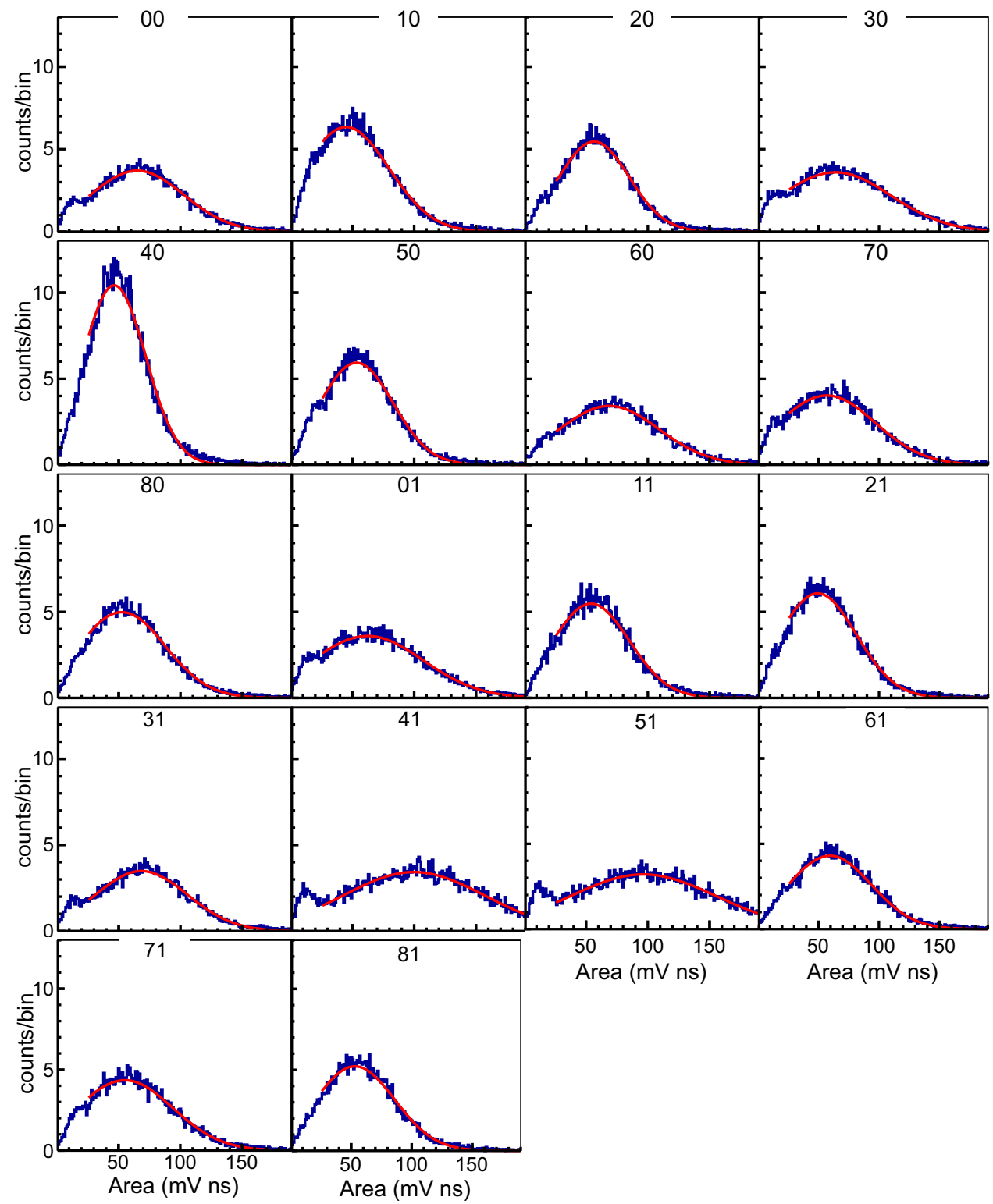


Table 3 Average of the light collected per unit of deposited energy by the nine $\mathrm{NaI}(\mathrm{Tl})$ modules composing ANAIS-112 setup, derived from ANAIS-112 first year data, and standard deviation of the population

\begin{tabular}{lll}
\hline Detector & Average light collected $(\mathrm{phe} / \mathrm{keV})$ & Standard deviation \\
\hline D0 & 14.532 & 0.102 \\
D1 & 14.745 & 0.169 \\
D2 & 14.506 & 0.104 \\
D3 & 14.453 & 0.109 \\
D4 & 14.483 & 0.090 \\
D5 & 14.572 & 0.158 \\
D6 & 12.707 & 0.104 \\
D7 & 14.743 & 0.137 \\
D8 & 15.994 & 0.076 \\
\hline
\end{tabular}

filtering down to the threshold and hence, reduce analysis uncertainties in the search for dark matter annual modulation. Light collection is being continuously monitored by using periodic external calibrations using ${ }^{109} \mathrm{Cd}$ and the SER derived from the dark matter run closer to every calibration run. Figure 8 shows the evolution of the light collected per module along the first year of ANAIS-112 data taking, as well as the corresponding values for the light collected at each PMT unit separately. It is worth remarking that all the modules show a highly stable total light collection along this first year of data taking, and all but D4 and D5 modules also show a highly stable light collection at each PMT. For D4 and D5 clear anticorrelation in the light collected at each PMT is evident in the first months of data taking. No explanation for this behaviour has been found up to date.

\subsection{ANAIS-112 data analysis and blinding strategy}

The DAQ software produces as output ROOT [32] files with all the available information directly read from the electronic modules, mainly the PMT's waveforms, QDCs values and clocks readouts (real time and live time) These files are then further analyzed in three subsequent levels:

- First level We calculate pulse parameters, such as baseline level and RMS, pulse area (our low energy estimator) and PSA parameters. We also calculate the time since the last muon veto and apply the peak-finding algorithm and store the number and position of the identified phe for each pulse.

- Second level In a second step we calibrate the energy response of every detector in two different regimes (low energy (LE), and high energy (HE)), as explained in Sect. 3. The final LE calibration is carried out every three dark matter runs $(\sim 1.5$ months $)$.

- Third level We optimize the pulse shape cuts and calculate their efficiency. LE variable for single hit (multiplicity 1) events is blinded in this level of analysis, but it is kept unblinded for multiple hits events, which are used to calculate event selection efficiencies, and to check the stability of the experiment, as commented in Sects. 5 and 7 , respectively.

We unblind $\sim 10 \%$ of the total statistics ( $~ 34$ days selected randomly from the whole first year of data taking amounting to 32.9 days live time) which we use for background assessment and tuning some of the procedures, while the rest of data remains blinded, until the moment to apply them the annual modulation analysis procedure.

\section{Energy calibration}

ANAIS-112 experiment is calibrated every two weeks using external ${ }^{109} \mathrm{Cd}$ sources. All the nine modules are simultaneously calibrated using a multi-source system built in a flexible wire in order to minimize down-time during the dark matter run. The system is designed to guarantee radon-free operation, with the wires moving along a sealed tube inside the shielding up to reach their position in front of the Mylar window (see lower panel of Fig. 1). The source plastic housing contains a certain amount of bromine, that under irradiation of the ${ }^{109} \mathrm{Cd}$ gammas produce a new calibration line in correspondence with the $\mathrm{K}$-shell $\mathrm{Br} \mathrm{X}$-rays.

Every calibration run lasts for about 3-4 h. The energy of every event is obtained from the pulse area of the sum of the digitized waveforms, $S_{\text {sum }}=\sum\left(S_{0}(t)+S_{1}(t)\right)$. In Fig. 9 we show the calibration spectra for the nine modules corresponding to one of those calibration runs, arbitrarily chosen. The three main lines used for calibration (11.9, 22.6 and $88.0 \mathrm{keV}$ ) are fitted to gaussians and shown in red in the figure. The second one is in fact the sum of several nearby X-rays with average energy of $22.6 \mathrm{keV}$. A MC simulation showed that this average is not affected by the photon absorption in the path from the ${ }^{109} \mathrm{Cd}$ source to the NaI crystal. For the $\mathrm{Br}$ line we take as nominal energy the average of the two more intense $\mathrm{K}_{\alpha}$ lines, resulting in $11.9 \mathrm{keV}$. These lines are used for monitoring gain stability and to correct any possible 
Fig. 8 Evolution of the light collected per unit of energy deposited for the nine $\mathrm{NaI}(\mathrm{Tl})$ modules along the first year of ANAIS-112 data taking. It is being monitored every two weeks, in average. Solid red dots correspond to the total light collected per module, black and cyan correspond to the PMT-0 and PMT-1 values, respectively. Colors referenced are available in the online version of the paper

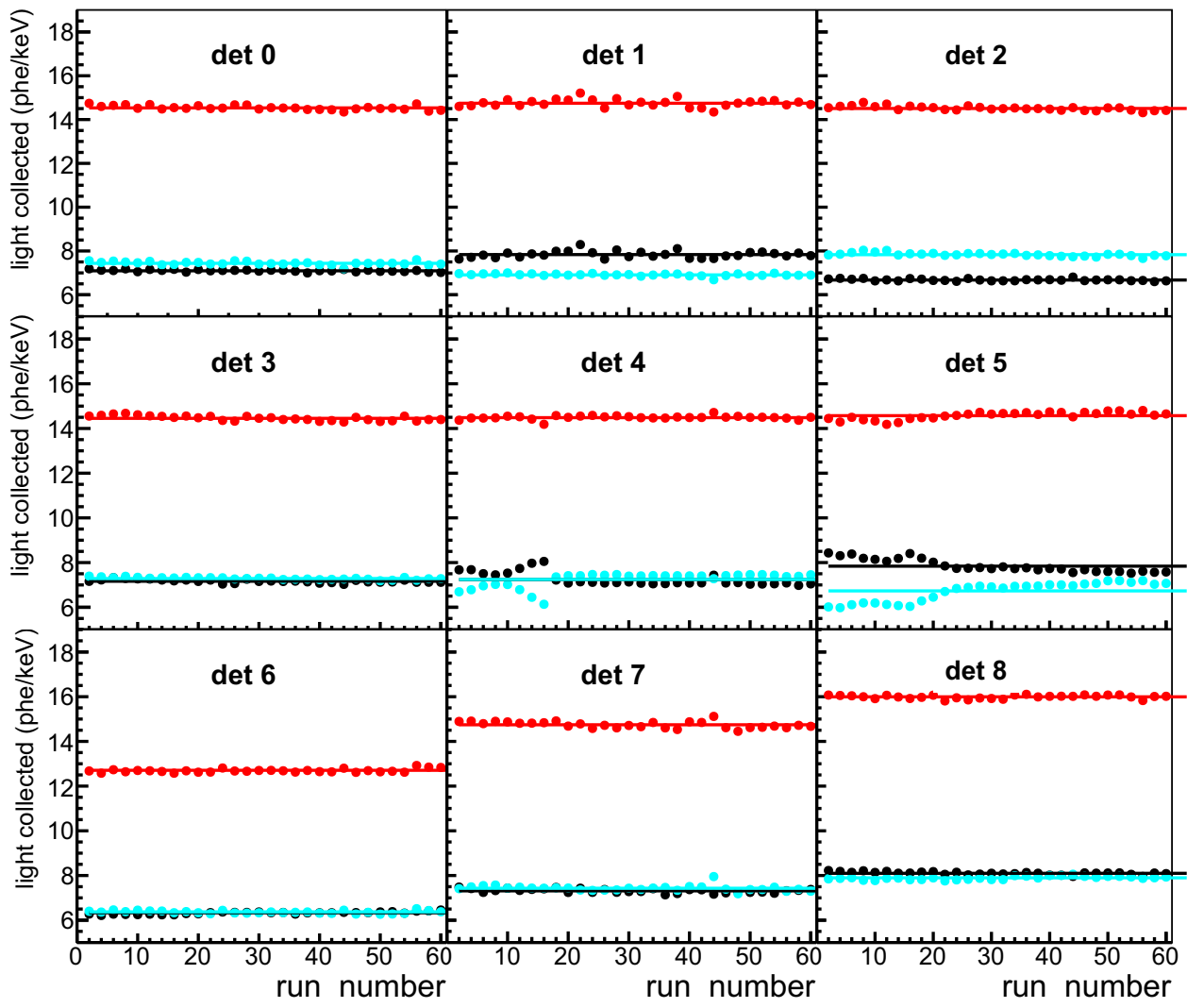

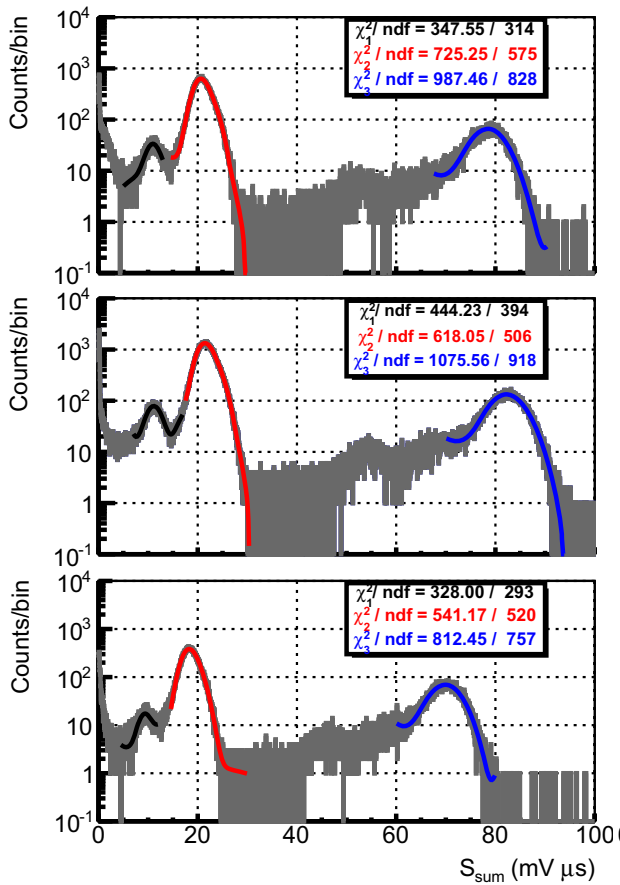

Fig. 9 Calibration spectra for the nine modules in one of the ANAIS112 calibration runs. The 11.9 and $88.0 \mathrm{keV}$ lines are fitted to single Gaussian lineshapes added to a second-order polynomial, while the $22.6 \mathrm{keV}$ line is fitted to two Gaussian lineshapes with the same standard deviation added to a first-order polynomial. All the three fits are
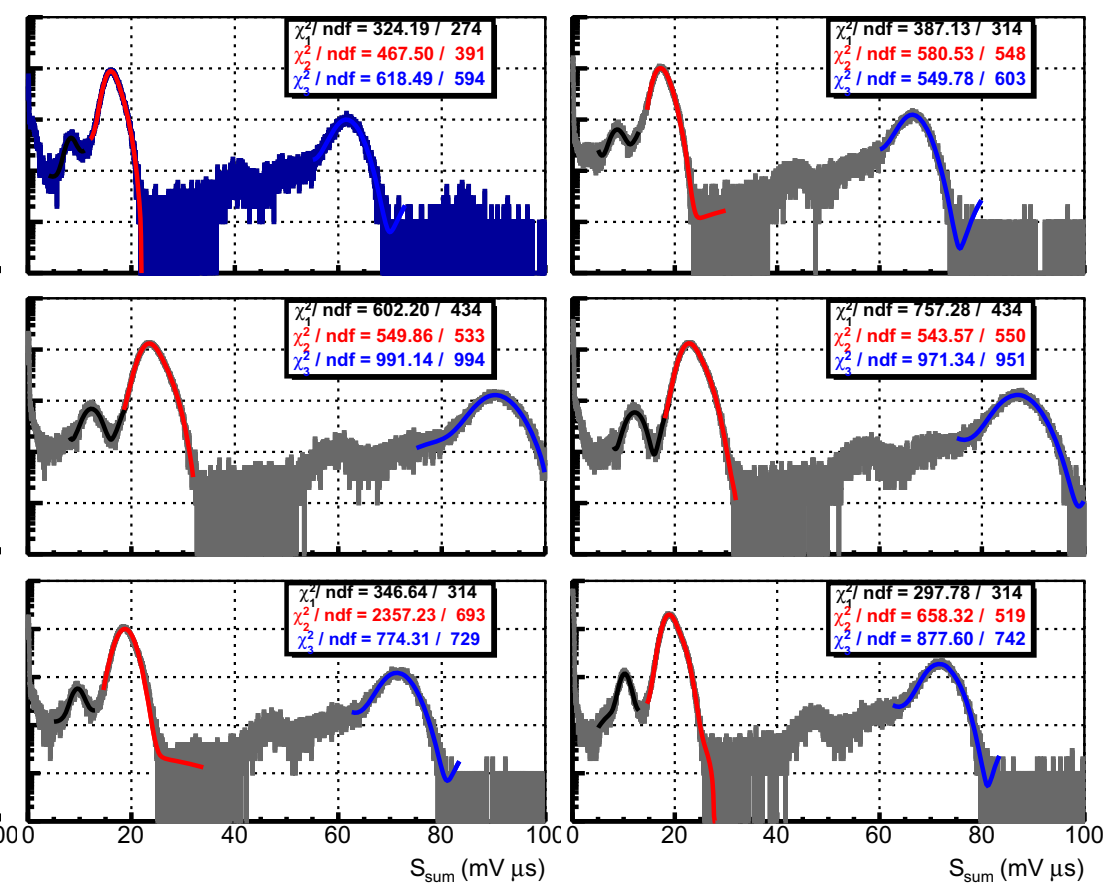

shown in red solid lines and chi-squared values for the three fits are given ( 1 - black, 2 - red, and 3 - blue labels correspond to the fits for $11.9,22.6$, and $88.0 \mathrm{keV}$ lines, respectively). Colors referenced are available in the online version of the paper 
Fig. 10 Mean positions of the three ${ }^{109} \mathrm{Cd}$ lines in the $S_{\text {sum }}$ variable along the first year of data taking for the nine ANAIS-112 modules. In blue $88.0 \mathrm{keV}$ line, in red $22.6 \mathrm{keV}$ line, and in black $11.9 \mathrm{keV}$ line. Only D4 and D5 have shown clear gain drifts related with the change in the sharing of light collection between the two PMTs (see Sect. 2). Colors referenced are available in the online version of the paper

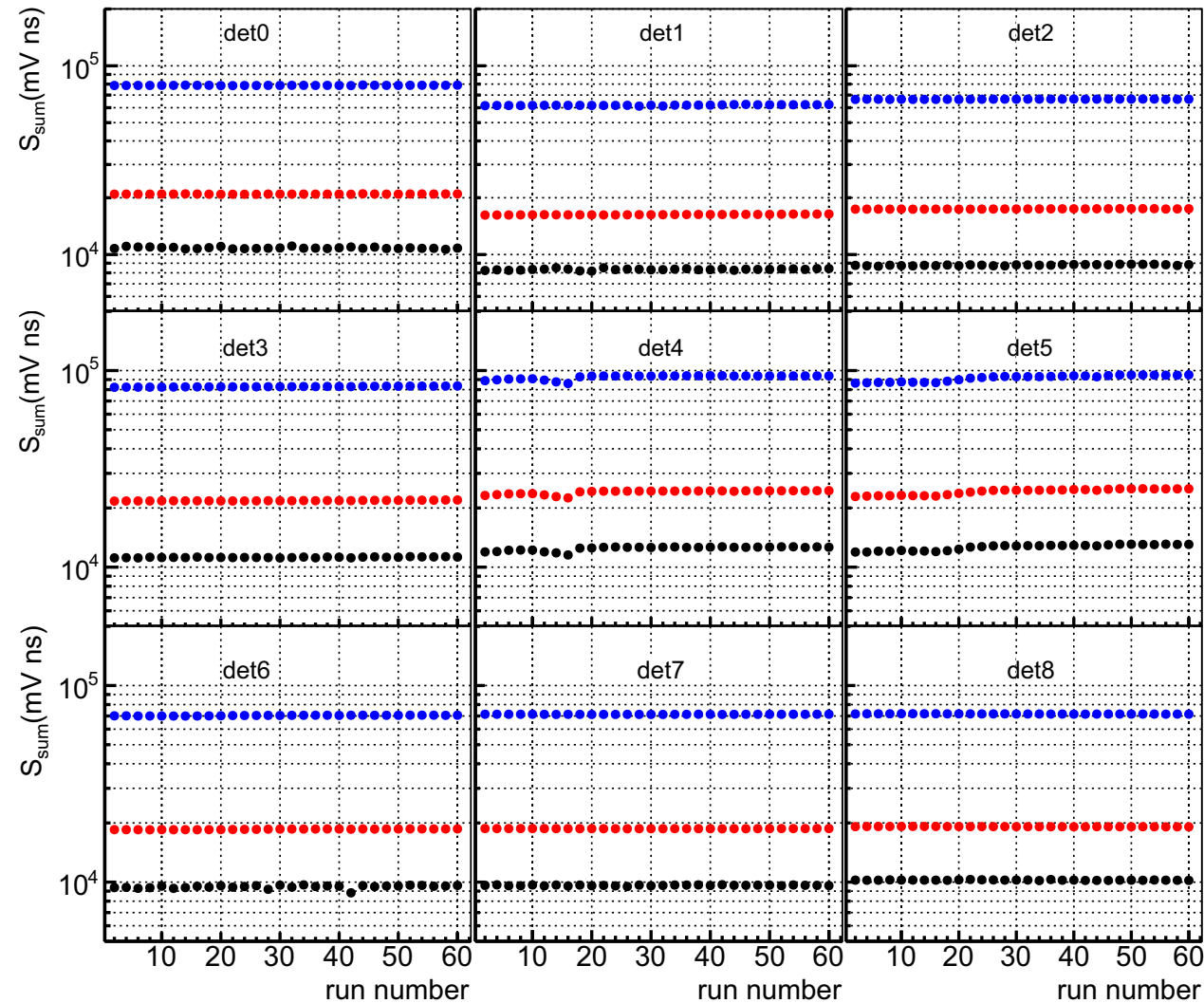

gain drift. In Fig. 10 we show the evolution of the positions of these three lines along the ANAIS-112 first year of data. Only D4 and D5 modules have shown clear gain drifts at the level of $10-15 \%$, related with the light sharing anomaly already shown and commented in Sect. 2.

Although the ROI for the annual modulation analysis is at very-low energy, below $10 \mathrm{keV}^{1}$ a good calibration in the high energy region is mandatory in order to verify the background model and properly select very relevant low energy events populations from coincidences with high energy gammas. Non-linearities in the detector response have been observed in ANAIS modules, being due to both, intrinsic non-linearities of the scintillator itself and PMTs saturation effects. Because of that, specific strategies for energy calibration have been applied to ANAIS data, both in the high and low energy regimes, as explained in Sects. 3.1 and 3.2.

\subsection{High energy calibration}

The pulse amplification is set to allow a good energy reconstruction below $10 \mathrm{keV}$, so the events saturate above $\sim 500 \mathrm{keV}$ in the digitized signal line. Because of that, the ANAIS-112 DAQ system (see Sect. 2.1) incorporated a second signal line conveniently attenuated, in order to keep information of the released energy of every high energy event using charge-to-digital converters for the signal instead of

${ }^{1}$ In the following, we will use $\mathrm{keV}$ for electron-equivalent energy. digitizers. In Fig. 11 the trend to saturation of the pulse area can be observed when is represented vs. the corresponding QDC value. These QDCs have a resolution of 4096 points, so, in order to reduce the quantization error in the energy signal, we do not take directly the QDC readout as estimator of the event energy in the high energy regime, but use this information to linearize the $S_{\text {sum }}$ response using a modified logistic function, as it is shown in Fig. 11 (green line). This linearized variable is used as estimator of the high energy assigned to each event above $\sim 50 \mathrm{keV}$. We also profit from the double readout system to discriminate $\alpha$ events (shown in red in Fig. 11). The integration window of both readouts is the same, but for high energy events the digitized pulses are saturated, so for the same QDC value the pulse integral is smaller for $\alpha$ events, as they are faster than $\beta / \gamma$ (shown in black).

Once the response is linearized, we calibrate the energy response above $\sim 50 \mathrm{keV}$ for each dark matter run independently using several peaks that are easily identified in the background, because no high-energy external sources are available for this purpose in ANAIS-112. For every detector and run we use between 6 and 8 peaks, that we fit to Gaussian lineshapes and use to perform a linear regression on their nominal energies against the Gaussian means using a second-order polynomial.

Figure 12 shows the calibrated anticoincidence (i.e., only single hits) background spectrum above $50 \mathrm{keV}$ for ANAIS112 in the whole exposure corresponding to the first year 


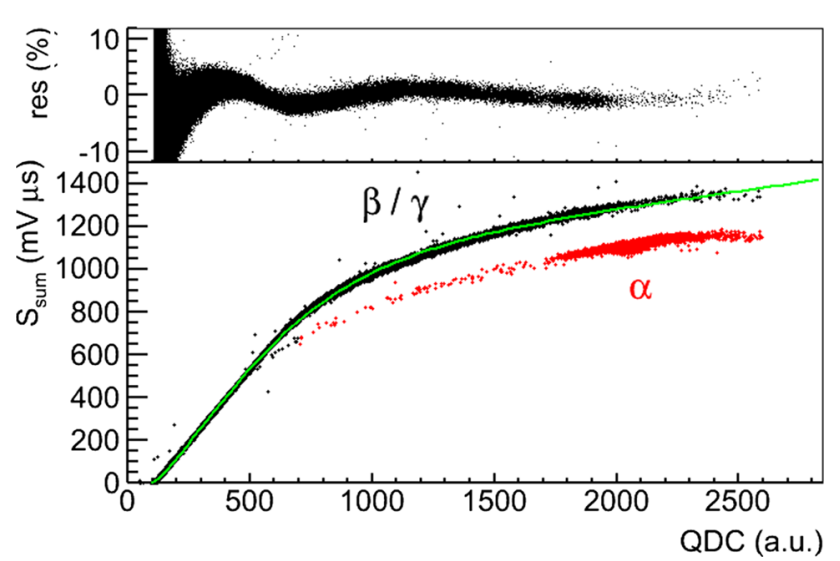

Fig. 11 Pulse summed-area of the digitized signals for one of the ANAIS-112 detectors during the two first weeks of data taking as a function of the QDC readout. Green line: modified logistic function used to linearize the $S_{\text {sum }}$ signal. Red dots: $\alpha$ population, clearly separated from the $\beta / \gamma$ one (black dots) due to the different $S_{\text {sum }} / \mathrm{QDC}$ ratio. The top panel shows the residuals of the $\beta / \gamma$ population fitting to the modified logistic function. Colors referenced are available in the online version of the paper

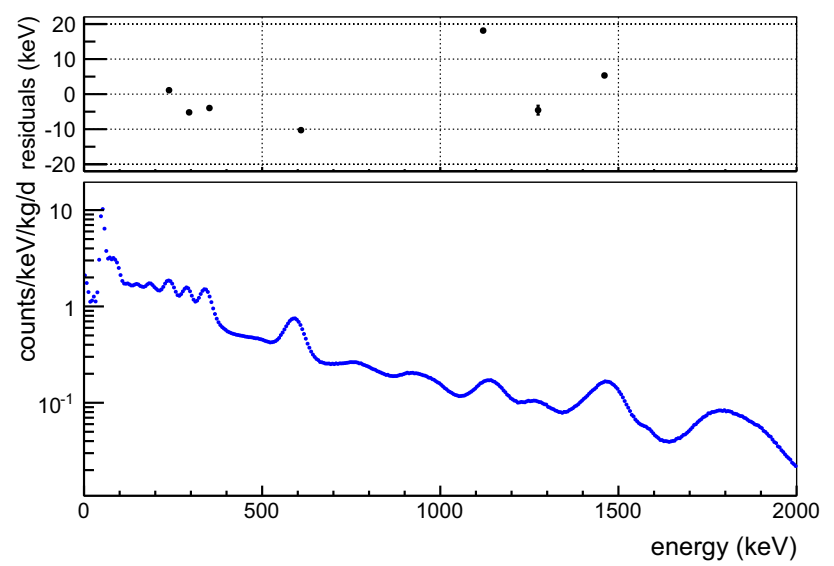

Fig. 12 Lower panel: high energy anticoincidence spectrum measured in the first year of ANAIS-112 data taking (all detectors). Upper panel: residuals (fit-nominal) for the positions of the main peaks identified in the background

of data taking. The upper panel shows the residuals for the positions of the main peaks identified in the background, being most of them below $10 \mathrm{keV}$.

\subsection{Low energy calibration}

Thanks to the Mylar window built in the ANAIS modules we have several calibration peaks from external sources very close to the ROI, but we can also profit from known lines present in the background to increase the calibration accuracy in the ROI. Because the two background lines considered are actually either in the ROI or very close to the threshold, and they come from energy depositions in the bulk (as dark matter is expected to do), their inclusion in the calibration procedure

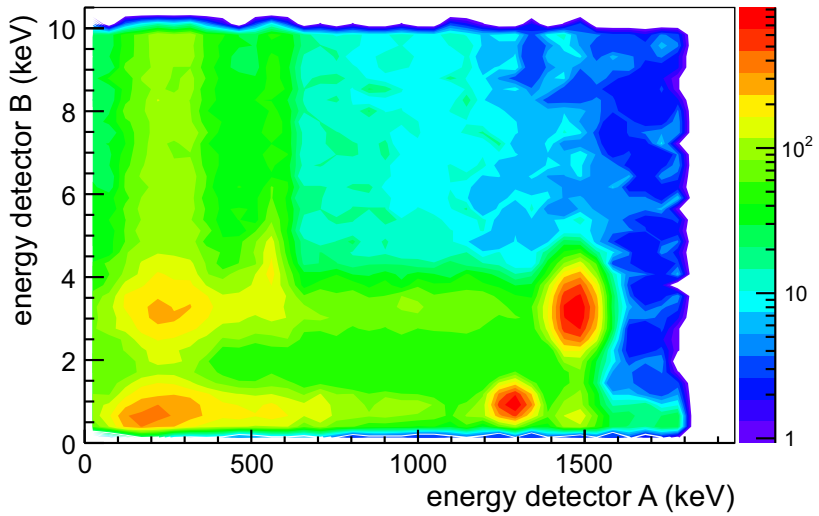

Fig. 13 Plot of the energies registered in two detectors triggered in coincidence when one of them has an energy deposition below $10 \mathrm{keV}$. Data correspond to the full first year of ANAIS-112. The spots at (1274.5 keV, $0.87 \mathrm{keV})$ and (1460.8 keV, $3.2 \mathrm{keV})$ correspond to Kshell EC decays from ${ }^{22} \mathrm{Na}$ and ${ }^{40} \mathrm{~K}$ in the NaI crystal, respectively

improves strongly the reliability of the ANAIS-112 energy threshold estimate. Figure 13 shows the energies registered in two detectors triggered in coincidence in the full first year of ANAIS-112. It shows those coincident events with one of the energy depositions below $10 \mathrm{keV}$. Two spots stand out in the picture, whose origin can be ascribed to ${ }^{22} \mathrm{Na}$ and ${ }^{40} \mathrm{~K}$ bulk contamination of the NaI crystals. These isotopes may decay via EC, with the emission of a $\gamma$ from the daughter nucleus de-excitation. The atomic de-excitation energy $\left(0.87 \mathrm{keV}\right.$ for ${ }^{22} \mathrm{Na}$ and $3.2 \mathrm{keV}$ for ${ }^{40} \mathrm{~K}$ for K-shell EC, which has the largest probability) is fully contained in the crystal where the decay occurs, while the high energy $\gamma$ (1274.5 and $1460.8 \mathrm{keV}$, respectively) can escape and hit another detector, thus producing a coincidence event. Compton-scattered $\gamma$ 's are also evident in the plot, with less prominent spots in correspondence with the backscattered events in the lead shielding (around $200 \mathrm{keV}$ ). These events can be taken as a population of background events homogeneously distributed in the NaI crystals array and falling in the energy window of interest for the annual modulation analysis, being then extremely interesting for evaluating any seasonal effect attributable to these backgrounds. Furthermore, they also serve as excellent calibration peaks. We use them to calibrate the ROI, together with the two lower energy lines recorded during the periodic calibration runs (11.9 and $22.6 \mathrm{keV})$. We calibrate with the external ${ }^{109} \mathrm{Cd}$ source the detectors every two weeks to control the gain stability and correct possible drifts, but in order to increase the statistics of the ${ }^{22} \mathrm{Na}$ and ${ }^{40} \mathrm{~K}$ peaks we add up one and a half months of dark matter runs to carry out the final LE calibration. It is worth mentioning that these peaks selected by the coincidence requirement are polluted with a population of very fast low-energy events in coincidence with a high-energy deposition in another crystal. They are presumably caused by an energy release in the PMT (likely an electron produced by a $\beta$ decaying isotope, which is able 

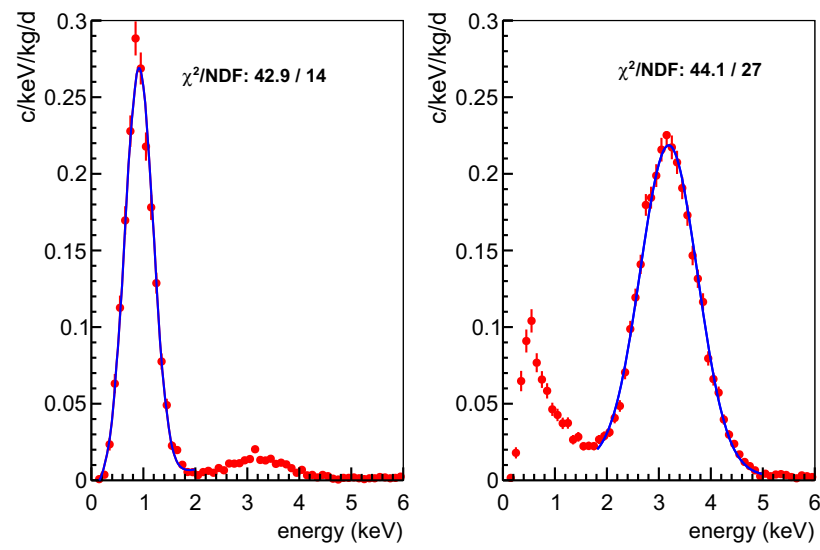

Fig. 14 Low energy spectra of the nine modules in coincidence with a high energy gamma in the range [1215-1335] keV (left panel) and [1350-1560] keV (right panel) in a second module (after recalibration described in Sect. 3.2). Data correspond to the full first year of ANAIS112. Peaks at 0.87 and $3.2 \mathrm{keV}$, attributed to ${ }^{22} \mathrm{Na}$ and ${ }^{40} \mathrm{~K}$ decay in the NaI bulk, respectively, can be clearly observed. Blue line: fit to a Gaussian plus linear background lineshape. The goodness of the fits is also shown in the plot. Colors referenced are available in the online version of the paper

to produce Cherenkov light) in coincidence with a $\gamma$ in one of the crystals. This hypothesis is further investigated in a companion paper [21]. As we will show in Sect. 4 they can be removed with a pulse-shape cut.

The peaks registered during calibration runs are fitted to Gaussian lineshapes, while for the ${ }^{22} \mathrm{Na}$ and ${ }^{40} \mathrm{~K}$ peaks, with a lower number of events, we take the median of the distribution. Then, we perform a linear regression on the expected energies against the peak's positions for every detector using a linear function and recalibrate the low energy events (below $50 \mathrm{keV}$ ).

We check the goodness of this procedure and the accuracy of the final calibration at LE precisely with the lines corresponding to ${ }^{22} \mathrm{Na}$ and ${ }^{40} \mathrm{~K}$ decays, found in the coincidence spectra of the whole available statistics for the first year of ANAIS-112. It is important to note that the $0.87 \mathrm{keV}$ peak from ${ }^{22} \mathrm{Na}$ is actually below our analysis threshold, established at $1 \mathrm{keV}$ after calculating the event selection efficiencies (see Sect. 5). However, as the events corresponding to this peak are selected by a coincidence requirement, they are almost free from noise/background except for the fast Cherenkov events mentioned above, so we can relax some of the cuts described in Sect. 4 and go beyond $1 \mathrm{keV}$ to fully reconstruct its energy after correcting by the trigger efficiency and that of the filter applied. More details on the selection cuts will be given in Sect. 5. We have followed this procedure to produce the low energy spectra shown in Fig. 14, in the left panel in coincidence with an energy deposition in the range [1215-1335] keV in another module (corresponding to the ${ }^{22} \mathrm{Na}$ population), and in the right panel requiring a coincidence with a [1350-1560] $\mathrm{keV}$ event $\left({ }^{40} \mathrm{~K}\right.$ popula-
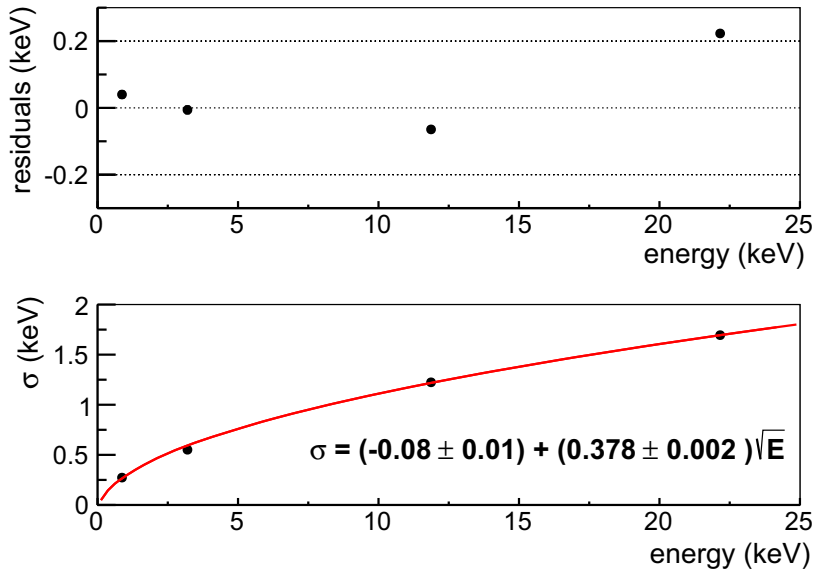

Fig. 15 Upper panel: residuals (fit-nominal) of the low energy calibration in the ${ }^{22} \mathrm{Na}$ and ${ }^{40} \mathrm{~K}$ background peaks (efficiency corrected) and the 11.9 and $22.6 \mathrm{keV}$ external calibration peaks. Lower panel: energy resolution of the same energy lines as a function of energy. The resolution at $22.6 \mathrm{keV}(11.9 \mathrm{keV})$ is calculated fitting the calibration peak to a combination of four (two) Gaussian lines. Red line corresponds to the fit to a $\alpha+\beta \sqrt{E}$ function, showing in the plot the values of the fitted parameters

tion). Two peaks at 0.87 (left panel) and $3.2 \mathrm{keV}$ (right panel) are clearly visible. The selection criteria for the ${ }^{22} \mathrm{Na}$ population includes also Compton-scattered $1460.8 \mathrm{keV}$ events, that produces the low-amplitude $3.2 \mathrm{keV}$ peak visible in the left panel. On the other hand, the asymmetric population at $\sim 0.5 \mathrm{keV}$ in the right panel could correspond to the L-shell EC decays from ${ }^{40} \mathrm{~K}$.

We fit both main peaks to a Gaussian lineshape plus a linear background (blue line). The residuals of the fit (defined as the difference between the reconstructed mean position of the peak minus the nominal energy) are shown in Fig. 15 together with those corresponding to the 11.9 and $22.6 \mathrm{keV}$ calibration peaks calculated in the spectrum obtained by adding all the calibration runs. According to this, the calibration deviations are below $0.2 \mathrm{keV}$ in the whole range and below $0.04 \mathrm{keV}$ in the ROI.

In the lower panel of Fig. 15 we show also the energy resolution as a function of energy calculated for the same lines ${ }^{2}$ in the coincidence spectrum and the addition of all the calibration runs, being very well reproduced by a $\alpha+\beta \sqrt{E}$ function. The $0.87 \mathrm{keV}$ peak, with a resolution of $\sigma=0.27 \mathrm{keV}$, is in good agreement with the expected value if the resolution is dominated by the phe counting statistics, considering an average light collection of $14.5 \mathrm{phe} / \mathrm{keV}$ (see Table 3).

\footnotetext{
${ }^{2}$ For the $22.6 \mathrm{keV}$ line we have used a four-Gaussian function shape, where all the Gaussians are constrained to have the same width and their relative intensities and positions are fixed to the four more intense $\mathrm{Ag}$ $\mathrm{X}$-rays [33], while for the $11.9 \mathrm{keV}$ line we have considered the same procedure with two Gaussians [34].
} 


\section{Event selection}

DM particles should interact with normal matter through processes having a very low cross-section. In general, they are typically searched for in the WIMP-nucleon cross-section range from $10^{-45}$ to $10^{-40} \mathrm{~cm}^{2}$ [3]. This implies that the probability of a dark matter particle interacting twice while crossing ANAIS-112 setup is negligible, and then we should only select events compatible with single-hit interactions, so coincidences among different modules are rejected. This very low interaction cross-section also means that the probability of interacting in the PMT or other setup components having low mass is also negligible, and that we should expect the interaction with the $\mathrm{Na}$ and I atoms as being dominant. Depending on the DM candidate, this interaction could occur only with the nuclei or also with the electrons in the target material. Because of this, we should remove from our analysis those events arising at the PMTs or being related with a muon crossing ANAIS-112 setup (see Sect. 2). In the following we summarize the filter procedures designed to select bulk scintillation events compatible with a dark matter signal and in next section the corresponding acceptance efficiencies for such filters will be calculated.

\subsection{Rejection of muon related events}

Figure 16 shows a detail on the trigger rate of ANAIS-112 dark matter run for a period of $70 \mathrm{~s}$. The time of every event triggering any of the ANAIS- 112 crystals is precisely determined using clock counters on a time basis of $50 \mathrm{~ns}$. Precise synchronization among clock counters in ANAIS-112 DAQ and Veto Systems is carried out periodically. Veto scintillators trigger timing is marked in Fig. 16 as dots on the upper axis, and it can be clearly seen the correlation with some of the remarkable increases in the total trigger rate of ANAIS112 modules. These high rate periods can then be related to a direct muon interaction in at least one of the $\mathrm{NaI}(\mathrm{Tl})$ crystals, which is producing subsequent triggers of the DAQ system. We remove most of these total trigger rate increases by rejecting events arriving less than one second after a veto scintillation trigger (see Fig. 16: blue line is total trigger rate, red line is the trigger rate after this filtering), and we correct off-line the live time of our measurement by subtracting to the live time one second for every muon veto trigger. Several experiments have measured a clear seasonal modulation in the muon rate underground, and we need to be sure that muon related events are conveniently rejected before the annual modulation analysis is applied. The fraction of live time rejected after the application of this filtering amounts only to a $3 \%$.

Unfortunately, we cannot reject all the muon related events in that way. Figure 17 top panel shows the total trigger rate in a period of $1000 \mathrm{~s}$, with a time binning of $0.1 \mathrm{~s}$. After one of

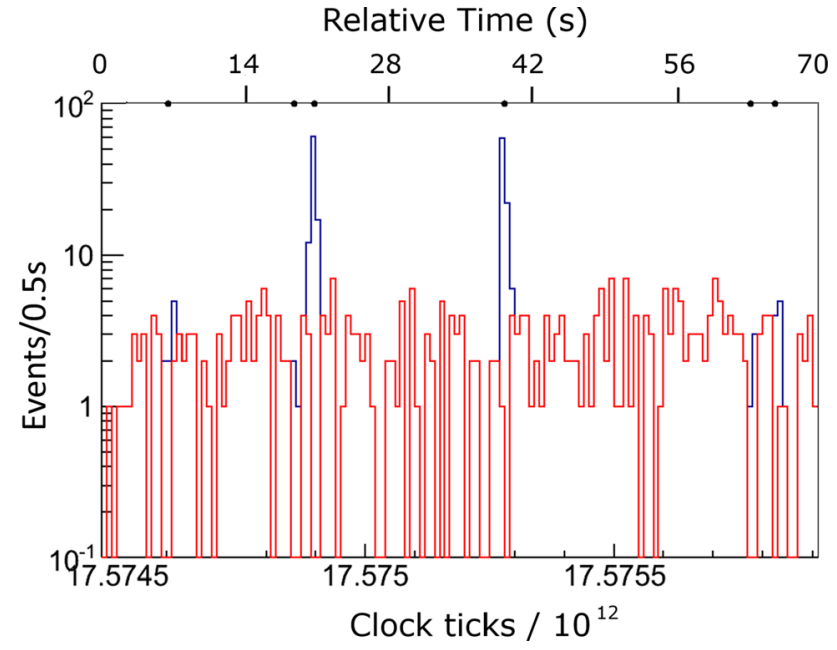

Fig. 16 Total trigger rate in ANAIS-112 in a period of $70 \mathrm{~s}$ is shown in blue. Events in the veto scintillators are marked as dots in the upper axis, pointing at a clear correlation between muons crossing ANAIS112 setup and total trigger rate increases. We show in red the trigger rate of events passing the filter of being more than one second after a trigger in the veto scintillators. Top axis time scale (given in seconds) is relative to the beginning of the period shown, bottom axis scale is relative to the beginning of the run and it is given in clock ticks ( $50 \mathrm{~ns} / \mathrm{tick})$. Colors referenced are available in the online version of the paper

the muons crossing ANAIS-112 set-up, identified by the Veto System, a very relevant increase in trigger rate is observed in the bottom panel of Fig. 17, which lasts for more than one second. Something similar is observed for very large energy depositions in one crystal and these events are not removed by the filter explained above. However, these events are easily eliminated by imposing an analysis threshold requiring more than one peak in the pulse at every PMT of a given ANAIS module. This analysis threshold is very soft, implying that at least 4 photoelectrons (phe) are required to produce an effective trigger ( 2 at each PMT). According to the light collection of ANAIS-112 modules reported in Sect. 2.4, it should not compromise being able to register events at $1 \mathrm{keV}$ or even below. Moreover, more stringent analysis threshold will be introduced in Sect. 4.2 and more information of the implications of this filter will be given there. In conclusion, we are quite sure we are removing muon related events from ANAIS-112 data. The energy distribution of the events removed using the $1 \mathrm{~s}$ after a muon filter is shown in Fig. 18 for the ANAIS-112 unblinded data (see Sect. 2.5). It is clear these events would contribute to the very low energy region if they were not rejected.

\subsection{Pulse shape cut}

In a scintillation detector there are events at low energy whose origin is neither the scintillating crystal, nor the PMT dark current, but other light-emitting mechanisms in the PMTs 

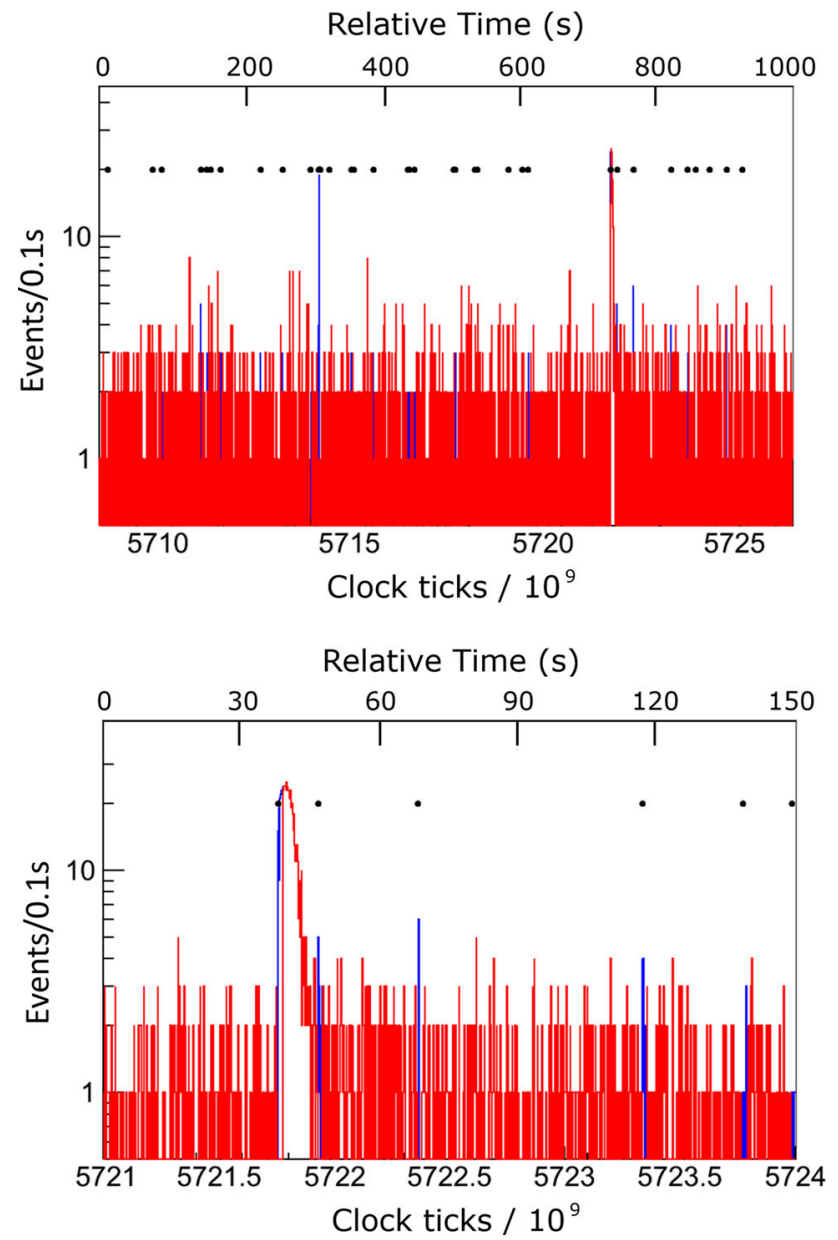

Fig. 17 Total trigger rate in ANAIS-112 in a $1000 \mathrm{~s}$ period is shown in blue and in red the trigger rate after removing $1 \mathrm{~s}$ following a veto trigger (top). A zoomed view around a very important muon related event is shown in the bottom panel. Some muon related events in the $\mathrm{NaI}(\mathrm{Tl})$ detectors are not removed by the $1 \mathrm{~s}$ delay filter, but those events have a very low number of phe in the pulses and they can be rejected too (see text). Top axis time scale (given in seconds) is relative to the beginning of the period shown, bottom axis scale is relative to the beginning of the run and it is given in clock ticks ( $50 \mathrm{~ns} / \mathrm{tick}$ ). Colors referenced are available in the online version of the paper

structure, the optical windows used to couple the crystal to the PMT, or even the optical grease or silicone pads used in that coupling. Unlike dark current events, which are effectively removed by the trigger in logical AND between the two PMTs, the latter events can dominate the trigger rate below $10 \mathrm{keV}$ and they have to be rejected prior to any dark matter analysis. On the other hand, slow scintillation components (up to several hundreds of $\mathrm{ms}$ ) have been measured in $\mathrm{NaI}(\mathrm{Tl}$ ) crystals after large energy depositions $(\alpha$ or $\beta / \gamma / \mu)$ [29]. These slow components can result in fake low energy events in the usual $\mathrm{NaI}(\mathrm{Tl})$ scintillation time scale if they are not properly identified as pulse tails.

The most common light production mechanism in the PMT is Cherenkov emission in the glass generated by

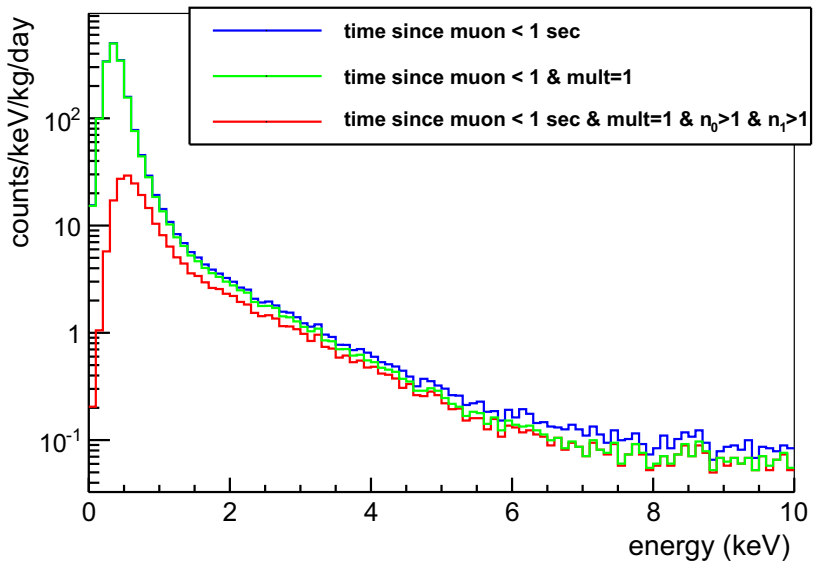

Fig. 18 Energy distribution (below $10 \mathrm{keV}$ ) of muon related events along the ANAIS-112 unblinded data: events occurring less than one second after a trigger of the Veto System (blue line), same but adding the anticoincidence condition (green line) and finally requiring also more than one phe at every PMT (red line). Colors referenced are available in the online version of the paper

radioactive contamination in the PMT itself or in the environment [28]. These events are very fast, in fact they should be dominated by the temporal behaviour of the PMT response (tens of ns range), and then, they should be easily discriminated by pulse-shape analysis (PSA) with respect to $\mathrm{NaI}(\mathrm{Tl})$ scintillation events (hundreds of ns range). For the sake of illustration we show in (a) panel of Fig. 19 a low energy bulk scintillation event from ${ }^{22} \mathrm{Na}$ decay and in panel (b) a fast pulse coming from the PMT. In order to discriminate this kind of events, we follow [35] and define the PSA parameter,

$\mathrm{P}_{1}=\frac{\sum_{\mathrm{t}=600 \mathrm{~ns}}^{\mathrm{t}=600 \mathrm{~s}}\left(\mathrm{~S}_{0}(\mathrm{t})+\mathrm{S}_{1}(\mathrm{t})\right)}{\sum_{\mathrm{t}=0}^{\mathrm{t}=600 \mathrm{~ns}}\left(\mathrm{~S}_{0}(\mathrm{t})+\mathrm{S}_{1}(\mathrm{t})\right)}$,

where $S_{0}(t)\left(S_{1}(t)\right)$ is the pulse amplitude at time $t$ after the trigger position for waveform $S_{0}\left(S_{1}\right)$. For $\mathrm{NaI}(\mathrm{Tl})$ scintillation pulses (dominated by the scintillation time of $\sim 230 \mathrm{~ns}$ ) this $\mathrm{P}_{1}$ parameter has a value around 0.65 , while for fast scintillation (Cherenkov-like) or single phe is usually below 0.2 .

This parameter also removes very efficiently random coincidences between individual dark current phe, however it is not useful to discriminate fake low energy events produced by long phosphorescence in the crystal or pulse tails (see Fig. 19 panel (c)). To achieve this scope we use the logarithm of the mean time of the distribution of the individual phe arrival times in the digitized window [16], $\log \left(\mu_{\mathrm{p}}\right)$, where $\mu_{p}$ is evaluated from the software trigger position until the end of the pulse as 
(a)

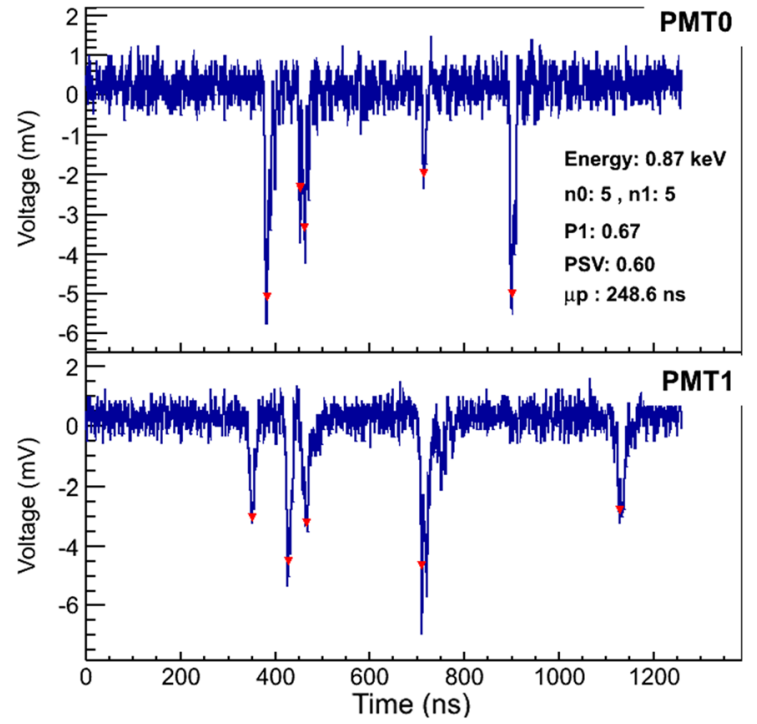

(c)

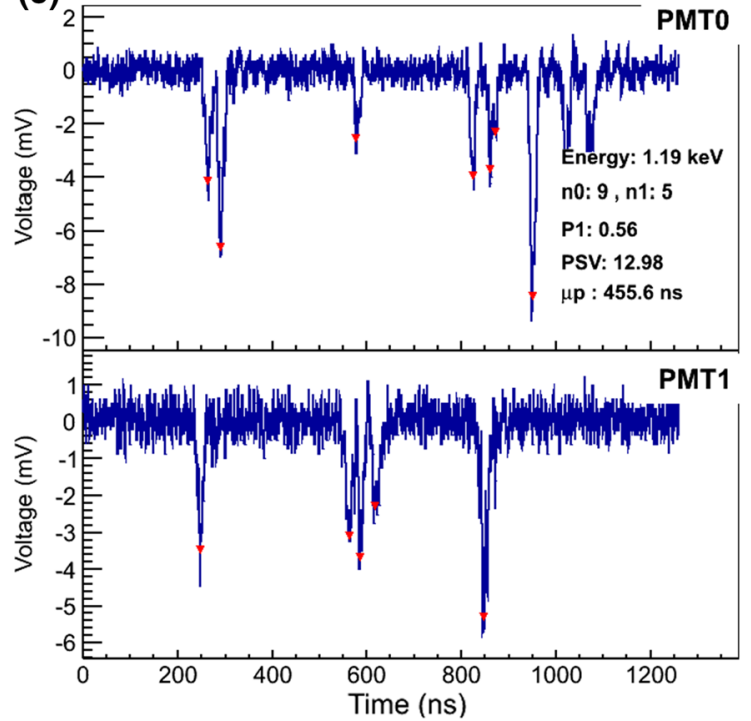

Fig. 19 Examples of low energy digitized pulses. The two traces at each panel correspond to the two PMT signals, while the legend display the energy of the event, number of peaks detected by the algorithm at every trace $\left(\mathrm{n}_{0}\right.$ and $\left.\mathrm{n}_{1}\right)$, and PSA parameters $\mathrm{P}_{1}$, PSV and $\mu_{p}$. Red triangles: phe identified by the peak-searching algorithm. a

$\mu_{p}=\frac{\sum_{p} A_{p} t_{p}}{\sum_{p} A_{p}}$

being $A_{p}$ and $t_{p}$ the amplitude and time of the phe identified in the pulse trace, respectively. For the phe identification we use the same peak identification algorithm developed for the SER construction (see Sect. 2.4).

In Fig. 20 we show the distribution of these shape parameters for the low energy events between [2-6] keV of detector $\mathrm{D} 1$ for the $\sim 10 \%$ unblinded data. Bulk scintillation events are clustered around $\left(\mathrm{P}_{1}=0.65, \log \left(\mu_{\mathrm{p}}\right)=-1.5\right)$, while (b)

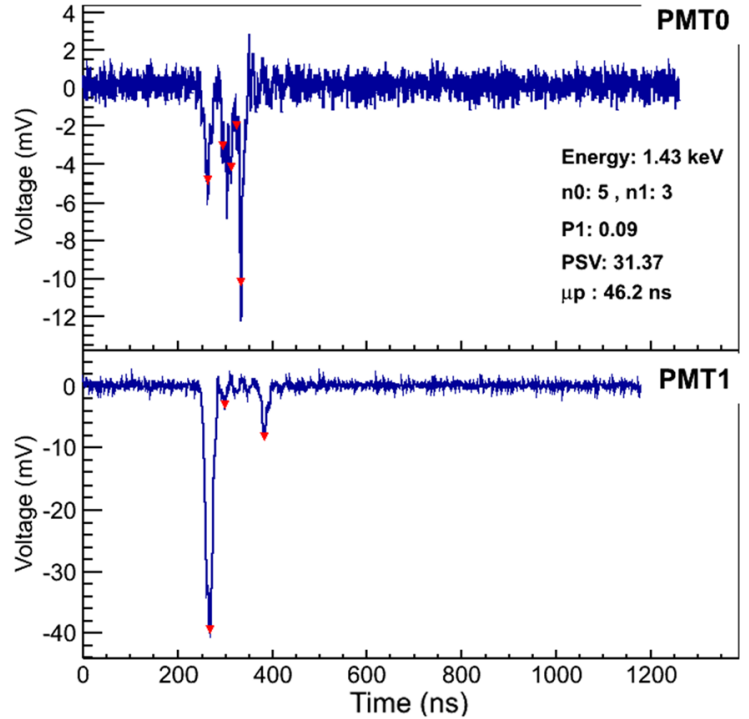

(d)

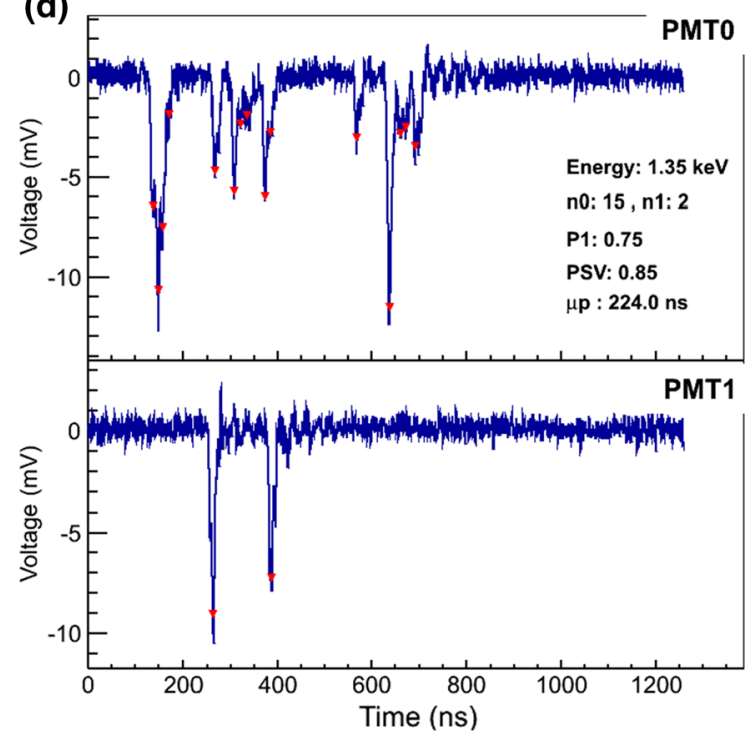

$0.87 \mathrm{keV}$ bulk scintillation event from ${ }^{22} \mathrm{Na}$ decay selected by coincidence with a $1274.5 \mathrm{keV}$ deposition in another crystal. b Fast event, likely a Cherenkov light emission in one PMT that is seen in the opposite PMT. c Long $\mu_{p}$ event, possibly caused by phosphorescence in the $\mathrm{NaI}(\mathrm{Tl})$ crystal. d Low energy asymmetric event

most background events at these energies correspond to very fast (likely Cherenkov) events with $\mathrm{P}_{1}<0.1$.

Both parameters are correlated, so we perform a bivariate analysis, assuming that $\boldsymbol{x}=\left(\begin{array}{c}\mathrm{P}_{1} \\ \log \left(\mu_{\mathrm{p}}\right)\end{array}\right)$ follows a 2dimensional Gaussian distribution with mean $\mu$ and covariance matrix $V . \mu$ and $V$ are calculated between [1-2] keV from the $0.87 \mathrm{keV}$ bulk scintillation population from ${ }^{22} \mathrm{Na}$ decay selected by coincidence with a $1274.5 \mathrm{keV}$ deposition in another crystal. We define,

$\operatorname{PSV}=(\mathrm{x}-\mu)^{\mathrm{T}} \mathrm{V}^{-1}(\mathrm{x}-\boldsymbol{\mu})$. 


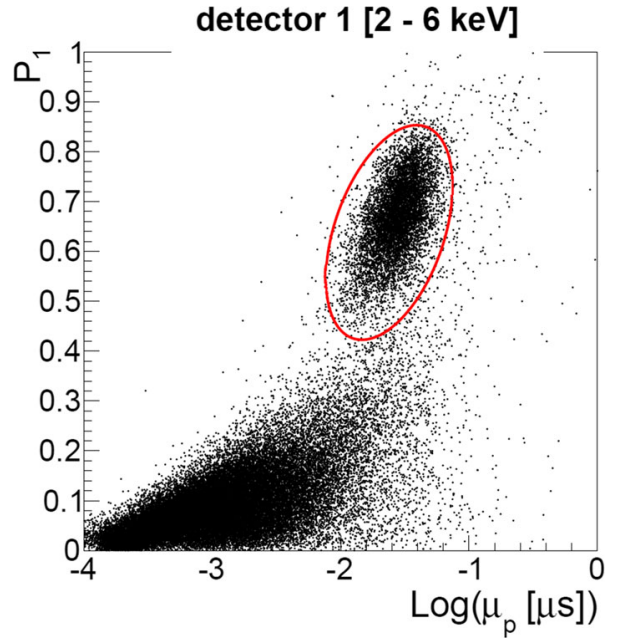

Fig. 20 Distribution of the background events of the $\sim 10 \%$ unblinded data of detector D1 with energies between 2 and $6 \mathrm{keV}$ in the $\left(\mathrm{P}_{1}, \log \left(\mu_{\mathrm{p}}\right)\right)$ plane. Bulk scintillation events are clustered around $(0.6,-1.5)$. The red line encloses the events selected by the PSV cut in this detector

For any given value of the parameter $\mathrm{PSV}_{c u t}$, the inequality $\mathrm{PSV}<\mathrm{PSV}_{\text {cut }}$ represents an ellipsoid centered at $\boldsymbol{\mu}$, and such that the probability that an event $\boldsymbol{x}$ lies outside the ellipsoid is given by $1-F_{\chi^{2}}\left(\mathrm{PSV}_{\text {cut }}\right)$, where $F_{\chi^{2}}$ is the cumulative $\chi^{2}$ distribution with 2 degrees of freedom [36]. We select $\mathrm{PSV}_{\text {cut }}=3$ (red line in Fig. 20), so the efficiency of the cut at [1-2] keV is $77.7 \%$, but as the distribution of the PSA parameters depends on energy, the efficiency of the cut in other energy regions has to be calculated, as we explain in detail in next section.

Below $2 \mathrm{keV}$, we observe a different population of background events whose main characteristic is a strong asymmetry in the energy partition among both PMTs (see an example in Fig. 19d). These events, which do not have the timing characteristics of afterpulses or pulse tails, are also observed by the COSINE collaboration [17], and could be related to the light emission observed in PMTs by other experiments $[37,38]$. They can be easily identified by the difference in the number of peaks that our algorithm finds in the signals of the two PMTs of every detector $\left(n_{0}\right.$ and $\left.n_{1}\right)$. In the panel (a) of Fig. 21 we show the distribution of $n_{0}$ and $n_{1}$ for background events corresponding to the $\sim 10 \%$ unblinded data with energies between 1 and $2 \mathrm{keV}$. We observe that most of them feature a very asymmetric distribution, with few peaks (one or two) in one PMT and more than five in the other one ${ }^{3}$. These events, only visible below $2 \mathrm{keV}$ in dark matter runs (see Fig. 21b), are not present neither in the ${ }^{109}$ Cd calibration runs (Fig. 21c), nor in the $0.87 \mathrm{keV}$ bulk scintillation popula-

\footnotetext{
${ }^{3}$ Note that, with more than 7 phe/keV/PMT, the probability of having 1 or 2 phe in one PMT at $1 \mathrm{keV}$ is around $3 \%$.
}

tion from ${ }^{22} \mathrm{Na}$ (Fig. 21d), so they are presumably fake light events produced at or near the PMTs.

This asymmetric population is not distinguishable from the bulk scintillation one by the PSV parameter, so to reject them we use a cut based on the number of peaks detected at every PMT: we require an event to have more than four peaks detected by our algorithm at every PMT signal (see red lines in Fig. 21). The efficiency of this cut is calculated on calibration runs as explained in next section.

\section{Efficiency}

We can factorize the total detection efficiency of ANAIS112 as the product of the trigger efficiency (probability that an event is triggered by the DAQ system), and the efficiency of the PSV and asymmetry cuts. For the muon vetoed events, we do not need an efficiency, but we calculate a $1 \mathrm{~s}$ dead time for every muon triggering the veto system, that is added to the total dead time of the DAQ system and then, discounted from the total live time. The efficiency of the anticoincidence cut can be safely taken as one, given that, with a total rate below $5 \mathrm{~Hz}$, the probability of an accidental coincidence between two detectors in the digitization window $(1.2 \mu \mathrm{s})$ is negligible.

\subsection{Trigger efficiency}

As explained in Sect. 2.1, the hardware threshold is low enough to efficiently trigger at the phe level each PMT. Nevertheless, at very low energy the coincidence requirement reduces the efficiency of the trigger, as very few phe are collected and the mean time interval between them increases.

For ANAIS-112 we do not expect a significant trigger efficiency reduction above $1 \mathrm{keV}$, given the high light collection and large coincidence window (200 ns). Trigger efficiency was experimentally measured for previous ANAIS prototypes in set-ups having two or three modules and a slightly different readout: all the modules traces were stored independently on the module triggering. ${ }^{22} \mathrm{Na}$ and ${ }^{40} \mathrm{~K}$ high energy events allowed to identify real coincidence events with a very low energy deposition in one of the modules, and by selecting those effectively triggering our DAQ to calculate the trigger efficiency [39]. Very high trigger efficiency was observed then, and as far as the same DAQ hardware/software is used in ANAIS-112, we do not expect differences in trigger efficiency in the present set-up. To quantify the trigger efficiency we use a Monte Carlo technique: for every detector we simulate $2 \times 10^{4}$ events of random energy $E$ between 0 and $10 \mathrm{keV}$. For each simulated event, the number of phe in a PMT is selected according to a Poisson distribution with mean given by $E \times \mathrm{LC}_{[\text {det }, P M T]}$, where $\mathrm{LC}_{[\operatorname{det}, P M T]}$ is the measured light collection for that detector and PMT (see 


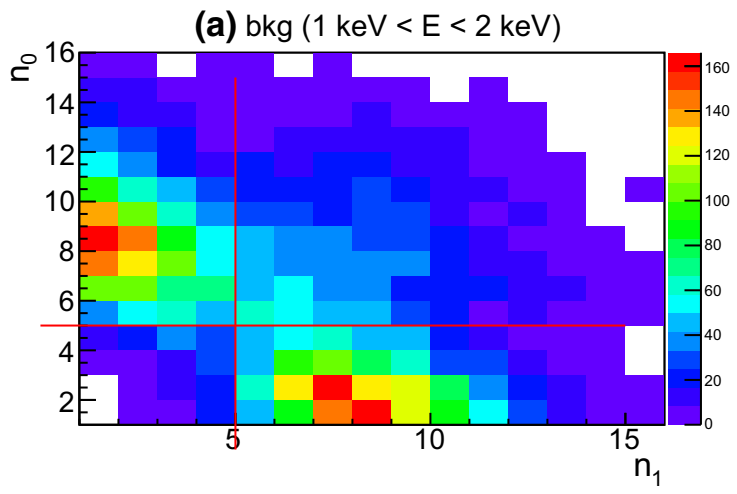

(c) calibration $(1 \mathrm{keV}<\mathrm{E}<2 \mathrm{keV})$

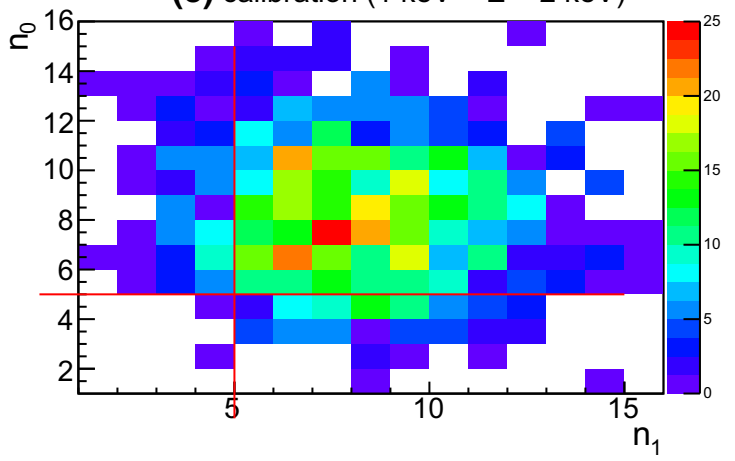

Fig. 21 Distribution in the $\left(\mathrm{n}_{0}, \mathrm{n}_{1}\right)$ plane (i.e., number of peaks detected by the algorithm for each of the two PMT signals from the same module) for: a $\sim 10 \%$ unblinded data background events with energy between 1 and $2 \mathrm{keV}$; $\mathbf{b}$ same but for events with energy between 2 and

Table 3). The phe are simulated as Gaussian peaks of widths between 5 and $6 \mathrm{~ns}$, depending on the detector. The arrival time of every phe is sampled from an exponential PDF with $\tau=230 \mathrm{~ns}$, as for $\mathrm{NaI}(\mathrm{Tl})$, and their amplitudes are sampled from the SER amplitude distribution of the corresponding PMT. Then, we calculate the trigger efficiency as a function of energy as the number of events having the first phe identified in every PMT above the trigger threshold and within $200 \mathrm{~ns}$ divided by the total number of simulated events generated for that energy. The result is displayed in Fig. 22. As expected, the efficiency is larger than $98 \%$ down to $1 \mathrm{keV}$. The small differences among the detectors are only due to the spread in light collection and phe width. This MC will be very useful in following sections to check the efficiencies of PSV and asymmetry cuts, which are more difficult to check experimentally than trigger efficiency and that could be more affected by systematics.

\subsection{PSV cut efficiency}

From Eq. 3, the definition of the PSV variable provides the efficiency for every cut value, but it is only valid in the energy region in which the means and covariances are calculated, (b) bkg $(2 \mathrm{keV}<\mathrm{E}<3 \mathrm{keV})$

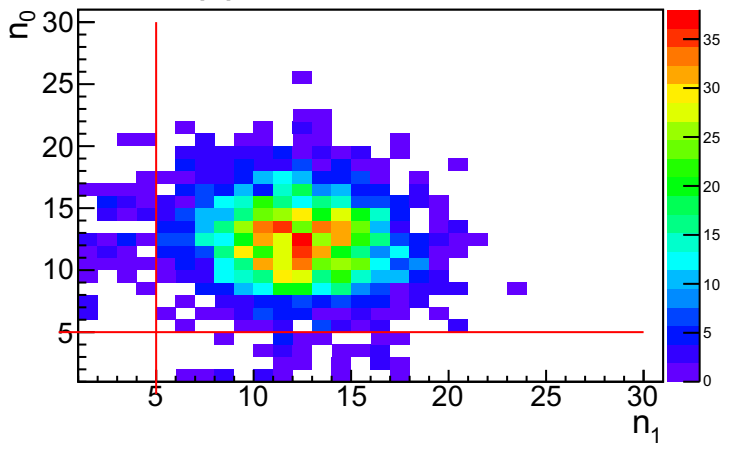

(d) bkg coin $1.3 \mathrm{MeV} \gamma(1 \mathrm{keV}<\mathrm{E}<2 \mathrm{keV})$

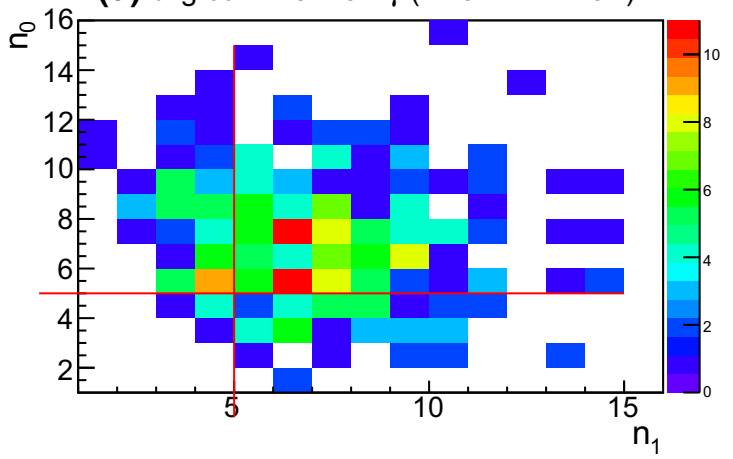

$3 \mathrm{keV}$; ${ }^{109} \mathrm{Cd}$ calibration events with energy between 1 and $2 \mathrm{keV}$; d bulk scintillation $0.87 \mathrm{keV}$ events from ${ }^{22} \mathrm{Na}$ decay selected by coincidence with a $1274.5 \mathrm{keV}$ deposition in another crystal (whole 1 year statistics)

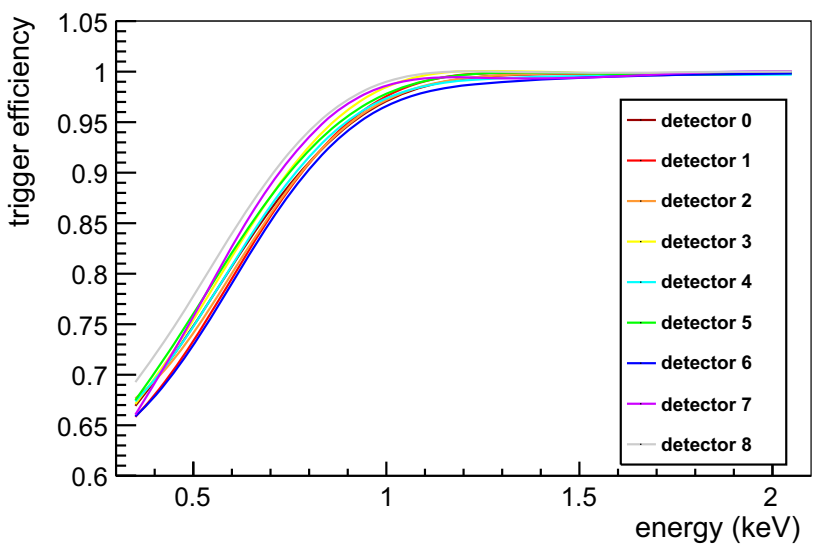

Fig. 22 Trigger efficiency for every detector, calculated from the measured light collected by a Monte Carlo technique. Colors referenced are available in the online version of the paper

i.e., between 1 and $2 \mathrm{keV}$. Instead of changing the variable definition for every energy range, we keep the same definition and calculate the efficiency of the cut as a function of energy. In order to do so we use the ${ }^{22} \mathrm{Na}$ and ${ }^{40} \mathrm{~K}$ low energy populations selected in coincidence with a $1274.5 \mathrm{keV}$ or $1460.8 \mathrm{keV} \gamma$ in another detector. 


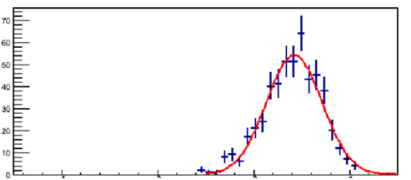

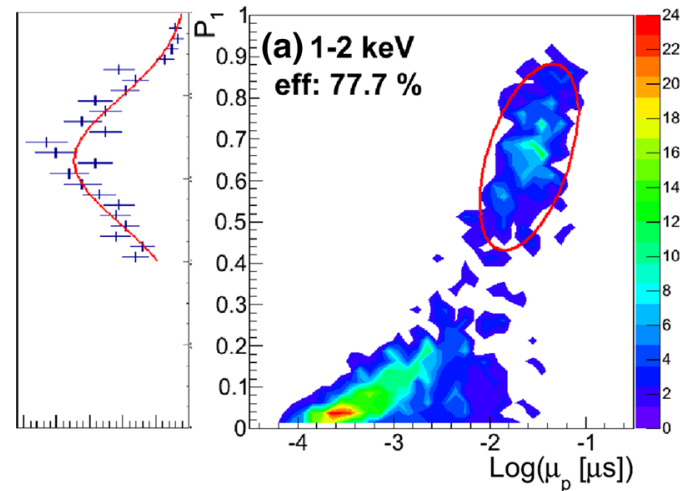

Fig. 23 Distribution of the ${ }^{22} \mathrm{Na} /{ }^{40} \mathrm{~K}$ events at low energy selected by coincidence with a $1274.5 \mathrm{keV}$ or $1460.8 \mathrm{keV} \gamma$ in another detector in the $\left(\mathrm{P}_{1}, \log \left(\mu_{\mathrm{p}}\right)\right)$ plane. The three panels correspond to $1-2,2-3$ and $3-4 \mathrm{keV}$ energy regions. The red line corresponds to PSV $=3$. The PSV variable is defined in the [1-2] keV range and the cut is fixed for an

Figure 23 shows the distribution of these low energy events of detector D1 in the PSA parameters plane for three energy ranges. In the first one (1-2 keV) we calculate $\mu$ and $V$ and fix $\mathrm{PSV}_{\text {cut }}=3$ (red ellipse) in order to have a $77.7 \%$ efficiency at that energy. This calculation is done for every detector independently. As we have commented in Sect. 3.2, the ${ }^{22} \mathrm{Na}$ and ${ }^{40} \mathrm{~K}$ populations are polluted with fast (Cherenkov-like) events in the PMT in coincidence with a $\gamma$ in one of the crystals [21]. Therefore, to avoid these events we require $\mathrm{P}_{1}>0.4$ before calculating $\boldsymbol{\mu}$ and $V$ (see projections along the $\mathrm{P}_{1}$ and $\log \left(\mu_{\mathrm{p}}\right)$ axis in Fig. 23a). Then, we compute the efficiency in $1 \mathrm{keV}$ bins for larger energies as the number of ${ }^{22} \mathrm{Na} /{ }^{40} \mathrm{~K}$ events in the ellipsoid divided by the total number of ${ }^{22} \mathrm{Na} /{ }^{40} \mathrm{~K}$ events having $\mathrm{P}_{1}>0.4$. Results are shown in Fig. 24 (continuous lines). Results are very similar for all the ANAIS-112 modules, with efficiency increasing from $77.7 \%$ at $1.5 \mathrm{keV}$ (fixed by the $\mathrm{PSV}_{\text {cut }}$ choice) up to $95 \%$ at $2.5 \mathrm{keV}$ and $98 \%$ at $3.5 \mathrm{keV}$. The statistical uncertainties in the efficiency, due to the small number of ${ }^{22} \mathrm{Na} /{ }^{40} \mathrm{~K}$ coincident events in each module, are of the order of $5 \%$ for all the modules. Although we calculate these uncertainties individually and propagate them conveniently to estimate the final uncertainties in the total efficiencies, they have been averaged to be shown in Fig. 24.

This procedure to estimate acceptance efficiencies is not valid for energies larger than $\sim 4 \mathrm{keV}$, as there are very few events selected by the coincidence condition (see Fig. 13). In order to extend the efficiency calculation up to $10 \mathrm{keV}$ and double-check the results, we use the MC simulation described in Sect. 5.1. To ensure consistency between the $\mathrm{MC}$ and the data, first we check that the distributions of the efficiency of $77.7 \%$ in that energy region. Then the efficiency is calculated for the other energy ranges as explained in the text. In a we show the projections of $\mathrm{P}_{1}$ and $\log \left(\mu_{\mathrm{p}}\right)$ variables in left and top panels, respectively, and the fitting to single Gaussian lineshapes, considering only $\mathrm{P}_{1}>0.4$ events, which supports the bivariate analysis carried out

PSA parameters are compatible. The distribution in $\log \left(\mu_{\mathrm{p}}\right)$ is shown in the inset of Fig. 24 (black dots), to be compared with the upper panel in Fig. 23. With the simulated events we follow the same method described before, i.e., we compute $\mu$ and $V$ between 1 and $2 \mathrm{keV}$ and construct the ellipse $\mathrm{PSV}=3$. Then we calculate the efficiency at higher energies as the number of events inside the ellipse divided by the total number of simulated events. The result is displayed in Fig. 24 as black closed dots, showing an excellent agreement with the efficiencies calculated on the ${ }^{22} \mathrm{Na} /{ }^{40} \mathrm{~K}$ populations.

Finally, it is worth noting that our sample population for the efficiency calculation is originated from electron recoils (ER), while for most DM models the expected signal comes from nuclear recoils (NR). It is well known that ER and NR feature slightly different scintillation constants in $\mathrm{NaI}(\mathrm{Tl})$ at very low energy. For example, in [16] the KIMs collaboration has measured a variation in the $\log \left(\mu_{\mathrm{p}}\right)$ parameter of 0.08 between the mean values for ER and NR at 2$4 \mathrm{keV}$, which roughly corresponds to a factor 0.9 in $\tau_{\text {scint }}$. We simulate a MC-NR population with a scintillation constant $\tau_{N R} \sim 0.9 \times \tau_{E R} \approx 205 \mathrm{~ns}$, and calculate the efficiency for the NR population when the PSV variable is computed on the ER one. Results are shown in Fig. 24 as open red squares. The largest difference in the efficiency is 0.03 in the region from 1 to $2 \mathrm{keV}$, that we will take as a systematic error in our efficiency estimate.

\subsection{Asymmetry cut efficiency}

We evaluate the efficiency of the asymmetry cut (more than 4 phe identified by the algorithm at every PMT) using all the 


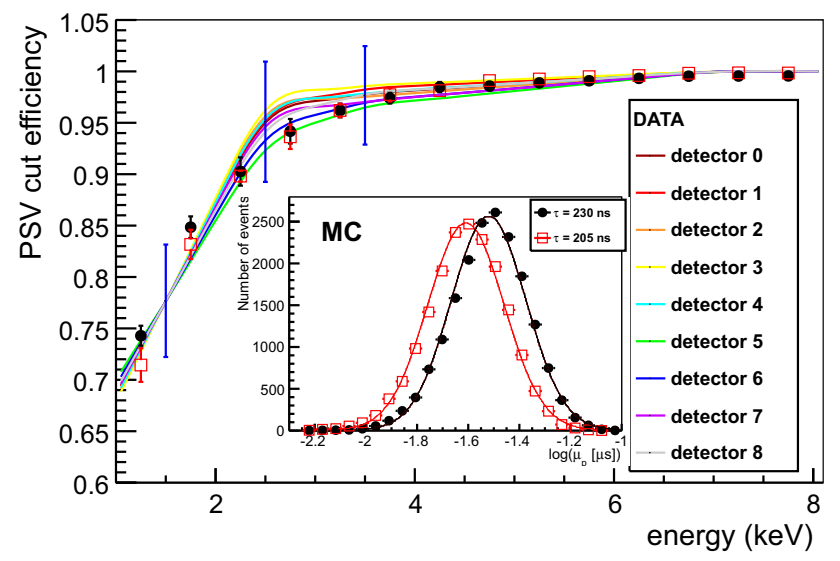

Fig. 24 Continuous lines: PSV cut efficiency for every detector, calculated from the distribution of the ${ }^{22} \mathrm{Na} /{ }^{40} \mathrm{~K}$ bulk events selected in coincidence with a high energy $\gamma$. The average of the statistical uncertainties for these efficiencies is shown in blue (see text for more information). Closed black dots: PSV cut efficiency calculated from a MC simulation of the scintillation pulses with $\tau=230 \mathrm{~ns}$. Red open squares: the same, but for a MC simulation with $\tau=205 \mathrm{~ns}$. Inset: distribution in $\log \left(\mu_{\mathrm{p}}\right)$ of the 3-4 keV events of both MC simulations. Colors referenced are available in the online version of the paper

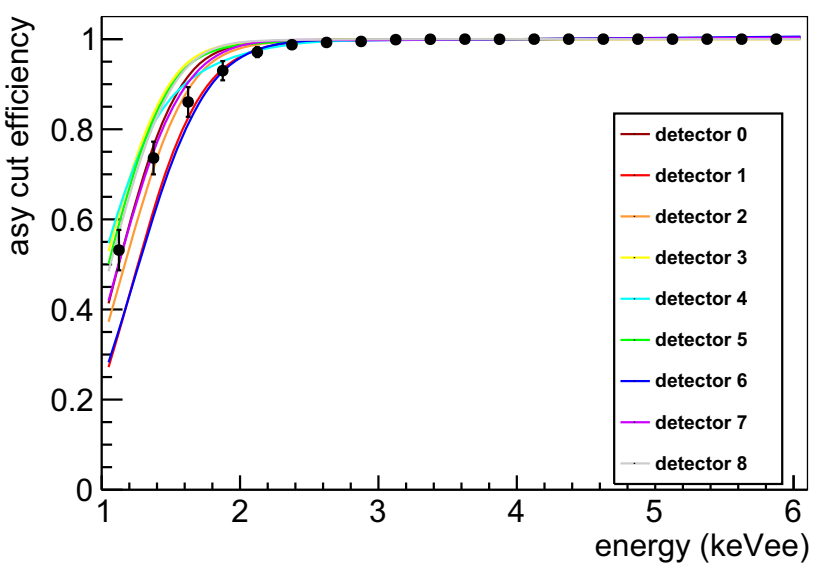

Fig. 25 Continuous lines: asymmetry cut efficiency for every detector, calculated in calibration runs. Black dots: the same, but calculated with the MC simulation. Colors referenced are available in the online version of the paper

${ }^{109} \mathrm{Cd}$ calibration events accumulated along the whole year calibration runs to have enough events in the $1-2 \mathrm{keV}$ energy region. In order to do so we count the number of events surviving the cut and divide by the total number of events, as a function of energy. Results are displayed in Fig. 25. The efficiency is equal to one down to $2 \mathrm{keV}$, and then it decreases steeply down to $0.3-0.55$ at $1 \mathrm{keV}$ (depending on the detector). We have chosen this energy, $1 \mathrm{keV}$, as common analysis threshold for all the modules, considering the acceptance efficiency of this filtering, which is the most restrictive. The result is in good agreement with expectations from Poisson statistics, taking into account that the peak-detecting algorithm cannot identify all the phe, as they overlap in the waveform. We have estimated the phe-finding efficiency of the algorithm with the MC simulation described in Sect. 5.1: it properly identifies between 70 and $80 \%$ of the phe at $1 \mathrm{keV}$, and then the efficiency decreases linearly with energy (as the probability of overlap increases) down to $40-50 \%$ at $5 \mathrm{keV}$. We show also in Fig. 25 the asymmetry cut efficiency calculated from the MC, averaged for all detectors (black dots).

We also check the consistency of the procedure with the ${ }^{22} \mathrm{Na}$ and ${ }^{40} \mathrm{~K}$ populations selected by coincidence. These events are not polluted with the asymmetric population, as we have shown in Fig. 21. Figure 26 compares for each detector the coincidence low energy spectra obtained after applying the PSV cut and correcting for the corresponding efficiency (red points) with the one obtained after applying also the asymmetry cut and correcting for the corresponding efficiency (black points). The agreement is excellent for all detectors down to $1.2 \mathrm{keV}$. Below this energy there is a discrepancy for some of the detectors (mainly D0, D1 and D6) up to $20 \%$ at $1 \mathrm{keV}$. We take into account this effect as a systematic error in the asymmetry cut efficiency.

\subsection{Total detection efficiency}

Finally we multiply the three efficiencies to obtain the total efficiency for the selection of dark matter compatible events in every ANAIS-112 detector, that is shown in Fig. 27. The estimated systematic errors in these efficiencies, previously commented, are shown in the width of the lines. At $1 \mathrm{keV}$ the efficiency ranges from 0.15 to 0.35 , depending on the detector, increases up to 0.8 at $2 \mathrm{keV}$ for all the ANAIS-112 modules and is nearly 1 at $4 \mathrm{keV}$.

\section{Low energy spectrum}

Figure 28 shows the energy spectra in the ROI corresponding to the $\sim 10 \%$ unblinded data for each of the detectors in anticoincidence (single hit events) after event selection and efficiency correction. A detailed analysis of the different background contributions, based on MC simulations of quantified radioactive contaminants, is reported in the companion paper [21]. The background in the ROI is dominated by the internal contamination of the $\mathrm{NaI}(\mathrm{Tl})$ crystals themselves, and varies depending on the detector as do their powder purification procedures, crystal growing protocols and starting time of underground storage. The main internal background contributions come from ${ }^{40} \mathrm{~K},{ }^{210} \mathrm{~Pb}$ (contaminants present in the starting powder, and presumably entering into the powder purification or growing procedures through ${ }^{222} \mathrm{Rn}$, respectively), and ${ }^{22} \mathrm{Na}$ and ${ }^{3} \mathrm{H}$ (having cosmogenic origin). In all detectors the ${ }^{40} \mathrm{~K}$ peak at $3.2 \mathrm{keV}$ is clearly visible. The background level at $2 \mathrm{keV}$ ranges from 2 to 5 counts $/ \mathrm{keV} / \mathrm{kg} /$ day, depending on the detector, and then 
Fig. 26 Low energy spectra of the nine modules in coincidence with a high energy gamma between 1215 and $1335 \mathrm{keV}$ in a second module. Data correspond to the full first year of ANAIS-112. Red points: Data selected by the PSV cut and corrected with the corresponding efficiency (PSV and trigger). Black points: In addition to the previous one, we apply the asymmetry cut and correct with the corresponding efficiency. Blue line: Gaussian fit to the red points. The chi-squared of the fit is also displayed. Colors referenced are available in the online version of the paper
Fig. 27 Total efficiency, obtained as the product of the trigger, pulse shape cut and asymmetry cut efficiencies. The statistical and systematic errors estimated for all these efficiencies, as described in Sect. 5, have been combined in quadrature and are shown in the width of the lines
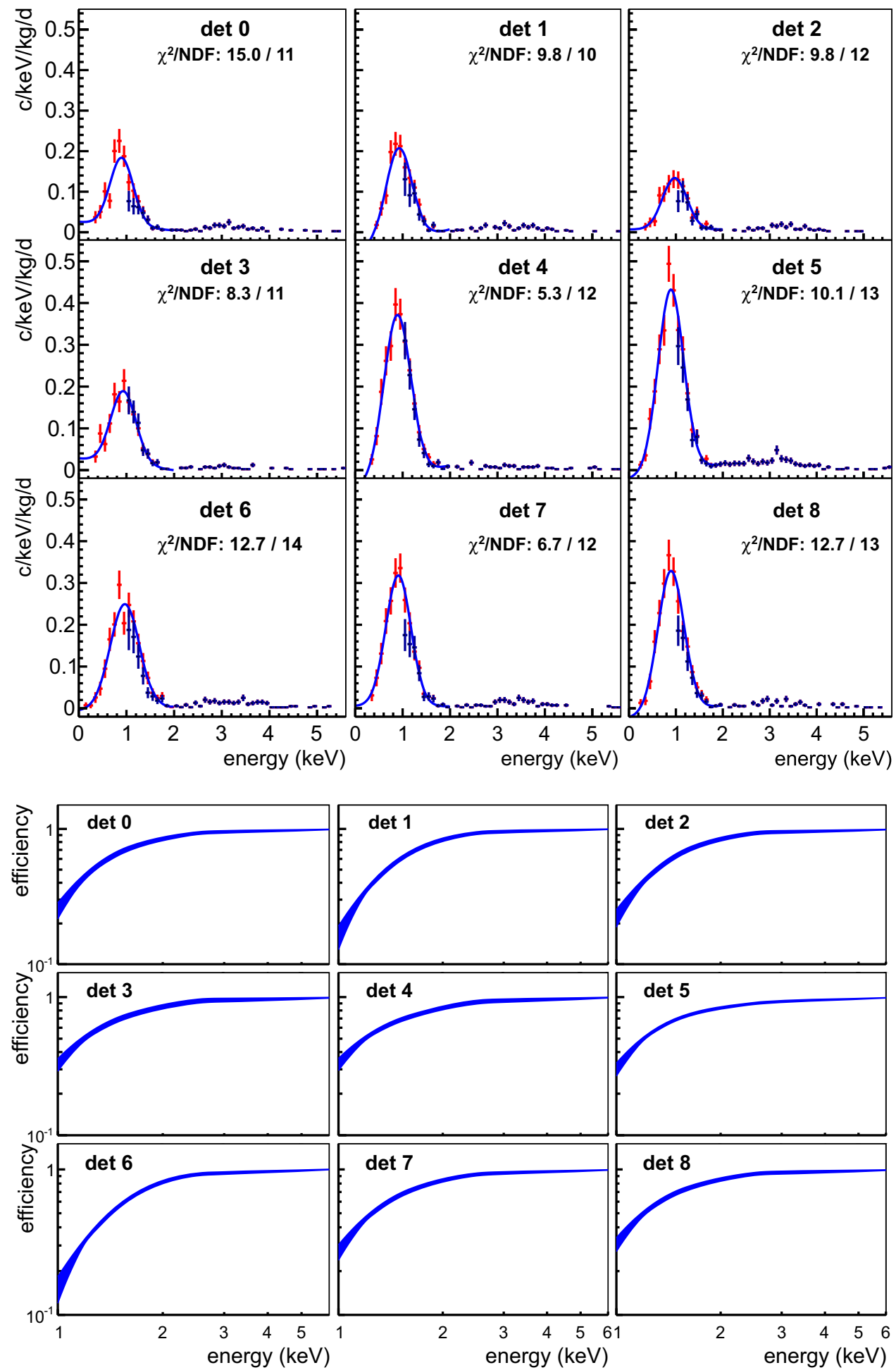

increases up to $5-8 \mathrm{c} / \mathrm{keV} / \mathrm{kg} /$ day at $1 \mathrm{keV}$. Corresponding values for the single-hit total spectrum of DAMA/LIBRA phase 2 are 1 and 1.8 counts $/ \mathrm{keV} / \mathrm{kg} /$ day, respectively [8].

We also display in Fig. 29 (blue points) the total anticoincidence background (adding up all 9 detectors) in the energy region below $100 \mathrm{keV}$ for the unblinded data. This energy region is also dominated by the internal contamina- tion of the crystal, with a prominent peak at $\sim 50 \mathrm{keV}$ coming from ${ }^{210} \mathrm{~Pb}$. The small peak at $\sim 26 \mathrm{keV}$ originated by ${ }^{109} \mathrm{Cd}$ and ${ }^{113}$ Sn cosmogenic isotopes $\left(T_{1 / 2}=461.9\right.$ and 115.1 days, respectively) is also visible. Above $100 \mathrm{keV}$ (see Fig. 12) the background is dominated by the PMTs radioactive contamination. In order to illustrate the magnitude of the rejected populations, we display in the same plot (Fig. 29 red points) 
Fig. 28 Anticoincidence energy spectrum measured in the ROI for each detector, corresponding to the $\sim 10 \%$ unblinded data. The ${ }^{40} \mathrm{~K}$ peak at $3.2 \mathrm{keV}$ is clearly visible

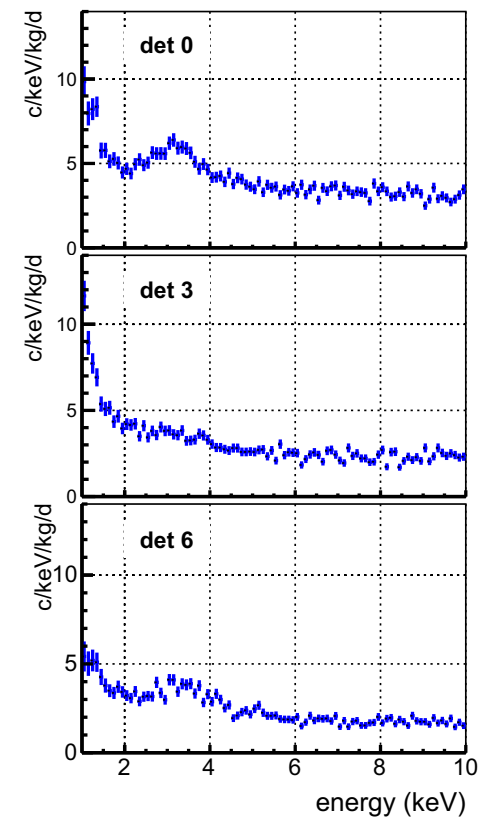

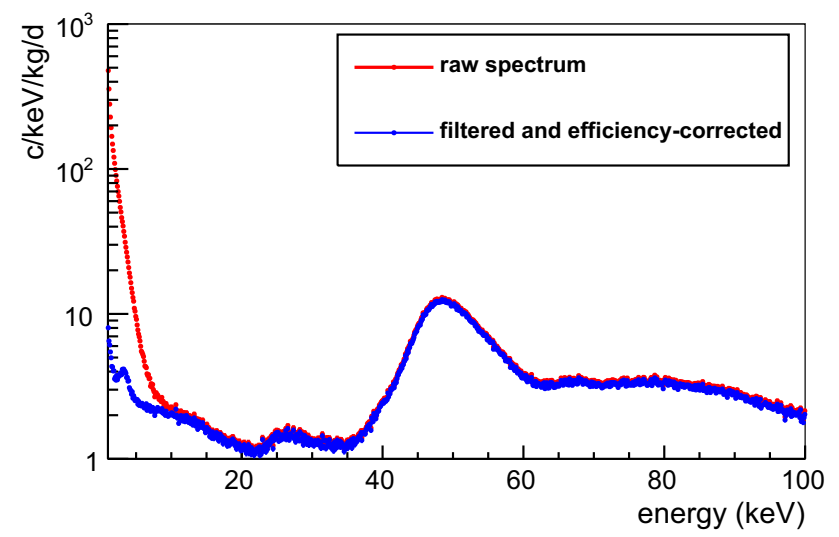

Fig. 29 Red points: total anticoincidence energy spectrum (all detectors) corresponding to the $\sim 10 \%$ unblinded data in the energy region from 0 to $100 \mathrm{keV}$. Blue points: same, but after applying the event selection criteria and correcting for efficiency. Main features identified in this spectrum correspond to the decay of isotopes found in the crystals: ${ }^{210} \mathrm{~Pb}$ (around $50 \mathrm{keV}$ ), ${ }^{109} \mathrm{Cd}$ and ${ }^{113} \mathrm{Sn}$ (from 20 to $30 \mathrm{keV}$ ) of cosmogenic origin, and ${ }^{40} \mathrm{~K}$ (around $3 \mathrm{keV}$ ). Colors referenced are available in the online version of the paper

the data before any filtering procedure (apart from the anticoincidence requirement). As we have commented before, non-bulk scintillation events clearly dominates the rate in the ROI and their correct identification is a key point in this kind of experiments.

\section{ANAIS-112 stability}

The annual modulation analysis requires to have under control all the environmental conditions which could produce any modulation in the detectors performance. Because of that, many of the environmental parameters that could affect ANAIS-112 setup have been monitored along this first year of data taking in order to evaluate the possible influence in any modulation which could be observed. In this section we also present an study of stability for several background events populations which can be taken as control samples in order to discard a possible relationship among a modulation in the ROI for dark matter analysis and the known sources of background.

Muon interaction rate onsite is being continuously monitored (see Sect. 2). Figure 30 shows the muon rate measured by each side of the Veto System (rate after PSA), and the muon coincidence rate between different sides, expressed both in muons/second, and calculated on a monthly basis. We had a problem with the QDC module used for the PSA required to identify muons in the scintillator vetoes, which produced a wrong identification of muons a few days in October-November 2017. Most of the data could be recovered, but for a few of the plastic scintillators, being noticed as an apparent decrease in muon rate in the East side of the scintillator Veto System at the end of October 2017.

Figure 31 shows the evolution of the rate of coincidences with high energy gammas in a second module attributed to the decay of ${ }^{40} \mathrm{~K}$ in the NaI bulk averaged every 10 days. As in Fig. 14, we apply the PSV cut and correct with the corresponding efficiency. The rate has been fitted to a constant rate (in blue) and to a straight line (in red). In both cases compatibility with constant rate is obtained. We have performed the same study with the ${ }^{22} \mathrm{Na}$ population selected by coincidence, which rate is expected to decrease with a half-life of 2.6 years. Figure 32 shows the evolution of the 

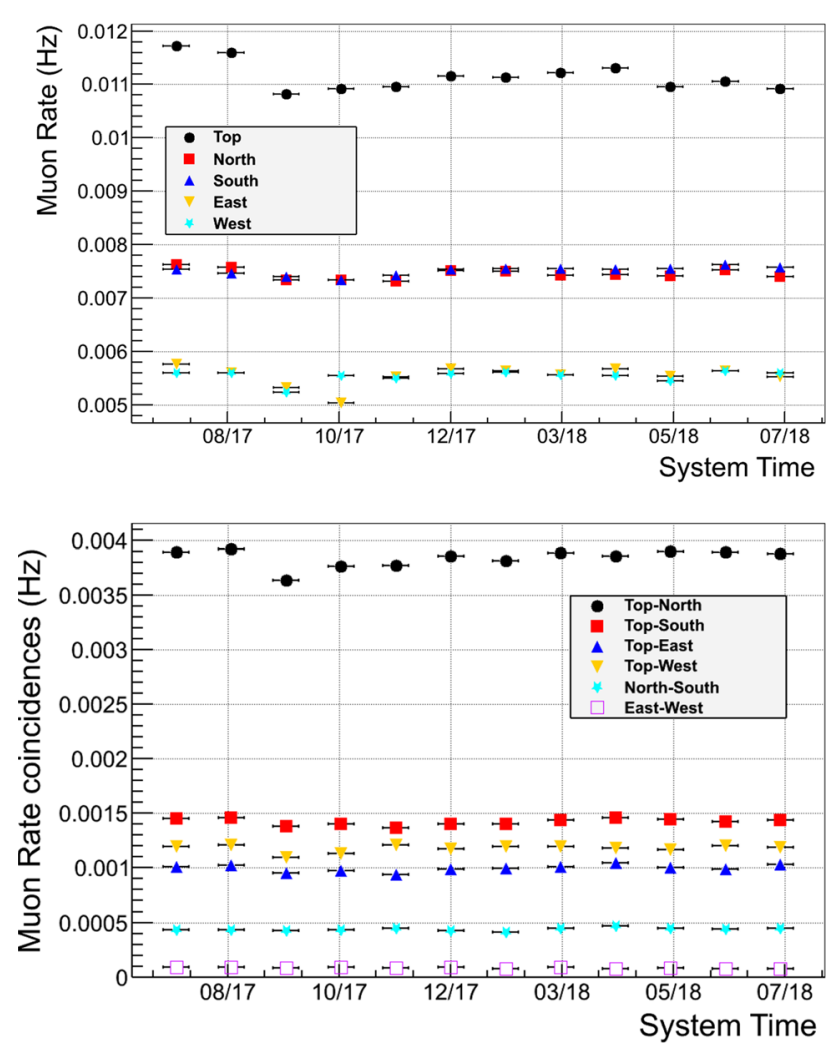

Fig. 30 Evolution of the muon rate. Upper panel: rate on each side of the scintillator veto system. Lower panel: muon coincidences rate between different sides of the scintillator Veto System. Rates are calculated per month. See text for more information. Colors referenced are available in the online version of the paper

natural logarithm of the ${ }^{22} \mathrm{Na}$ events selected by the coincidence requirement, with the same filtering as the ${ }^{40} \mathrm{~K}$ population. We fit to a straight line, obtaining a decay constant of $(0.00076 \pm 0.00014)$ days $^{-1}$ corresponding to a half life of $2.5 \pm 0.4$ years. These populations of events in the ROI would allow in the long term to check that the efficiencies of our filtering are stable or they show some modulation.

In Fig. 33 we show the rate of events in every module which have an energy deposition below $10 \mathrm{keV}$, pass the filtering procedure explained in Sect. 4, and are in coincidence with any energy deposition in a second ANAIS-112 module. Coincident events are defined as those triggering the ANAIS112 DAQ system in $1 \mu$ s window after the first trigger (see Sect. 2.1).

Figure 34 shows the evolution of the alpha specific activity derived from the rate of alpha events identified by PSA in every module along the first year of ANAIS-112. It can be observed that in D0 and D1 modules (which arrived in 2012 at the LSC), this activity is clearly decreasing with the expected ${ }^{210} \mathrm{~Pb}$ progenitor half-life, whereas in the case of the rest of modules the alpha rate is either stable or still slightly increasing, as ${ }^{210} \mathrm{Po}$ activity is still being built before reaching equilibrium in the decay chain.

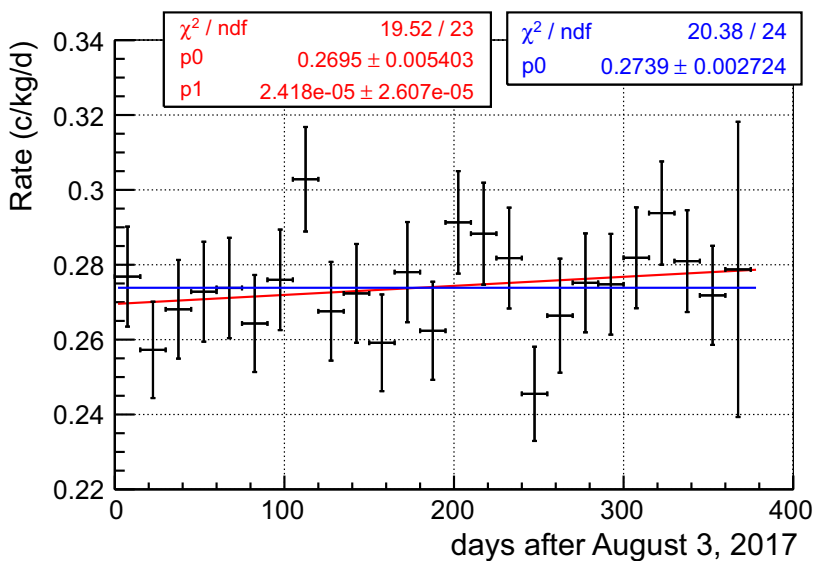

Fig. 31 Evolution of the rate of coincidences with a high energy gamma between 1400 and $1510 \mathrm{keV}$ in a second module, after filtering (PSV only) and efficiency correction in the energy range from 2 to $5 \mathrm{keV}$, corresponding to events attributed to ${ }^{40} \mathrm{~K}$ decays in the crystal bulk. Rates are averaged every 10 days and fitted to a constant rate (blue) or to a straight line (red). The results of the fits are also shown in the plot with the corresponding color. In both cases compatibility with constant rate is obtained. Colors referenced are available in the online version of the paper

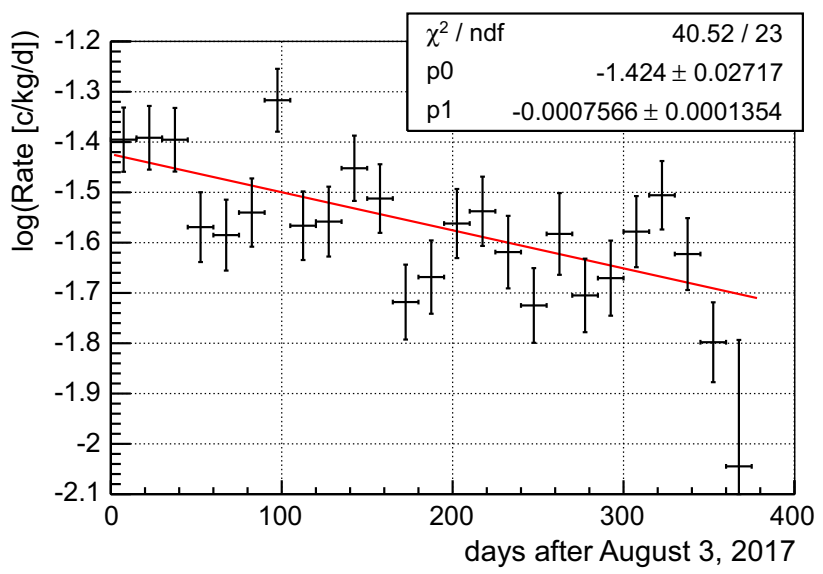

Fig. 32 Evolution of the natural logarithm of the rate of coincidences with a high energy gamma between 1215 and $1335 \mathrm{keV}$ in a second module, after filtering (PSV only) and efficiency correction in the energy range below $2 \mathrm{keV}$ corresponding to events attributed to ${ }^{22} \mathrm{Na}$ decays in the crystal bulk $\left(\mathrm{T}_{1 / 2}=2.6\right.$ years). Rates are averaged every 10 days and fitted to a straight line (red). The events rate is decreasing as expected with a half life of $2.5 \pm 0.4$ years

Figure 35 shows the evolution of the rate of events attributed to gamma background in three different energy windows. It has to be commented that D6, D7 and D8 have still cosmogenic activated isotopes decaying in the crystal bulk, because they arrived later at LSC. This can be clearly observed in Fig. 35.

Monitoring of environmental parameters has been continuously ongoing along ANAIS-112 dark matter run data taking: $\mathrm{N}_{2}$ gas flux entering into the shielding; temperatures at electronics, inner shielding, ANAIS hut, preamplifiers, etc.; 

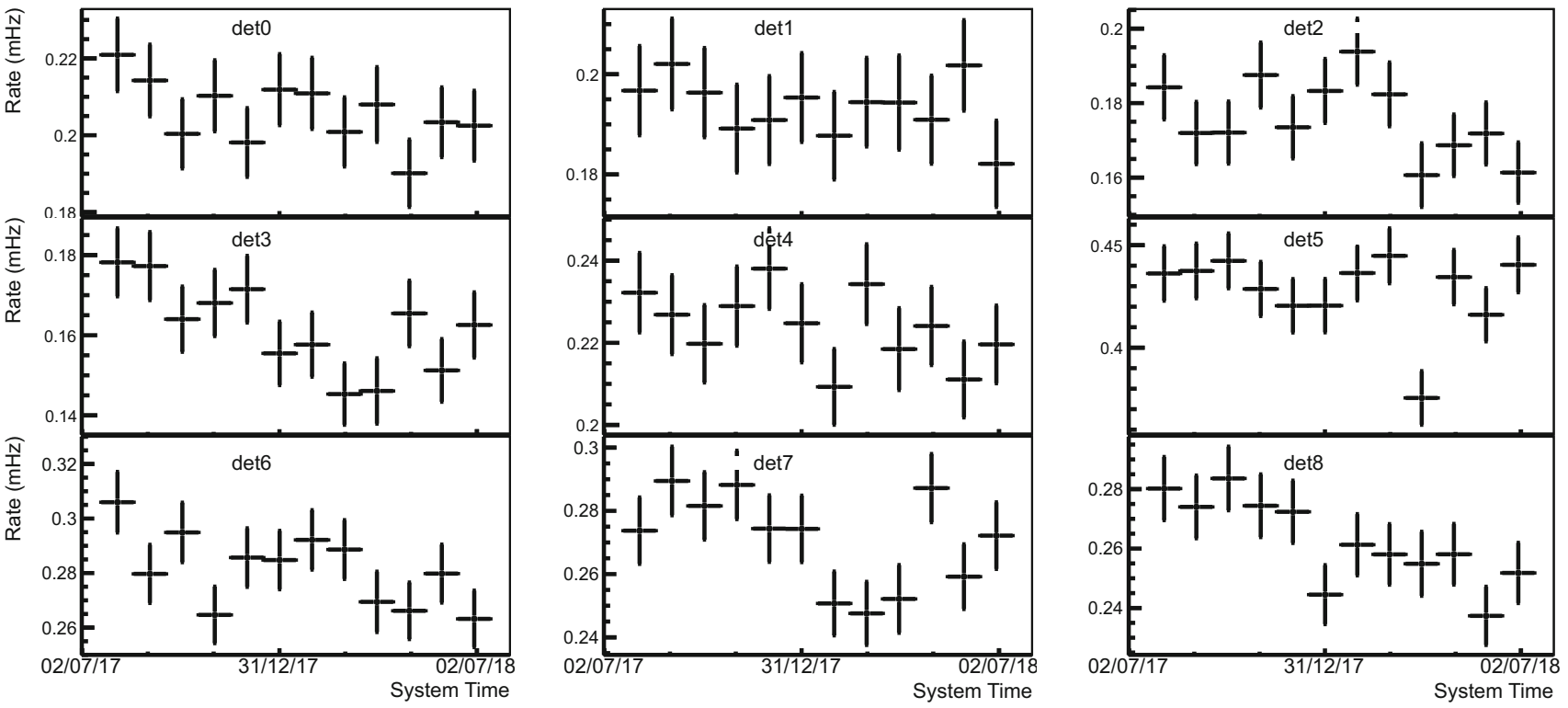

Fig. 33 Evolution of the rate of events producing an energy deposition below $10 \mathrm{keV}$ in the detector studied and passing the filtering procedure described in Sect. 4, which are in coincidence with any energy depo- sition in a second ANAIS-112 module. Coincident events are those triggering the DAQ system in $1 \mu \mathrm{s}$ window after the first trigger. Rates are calculated per month. See text for more information
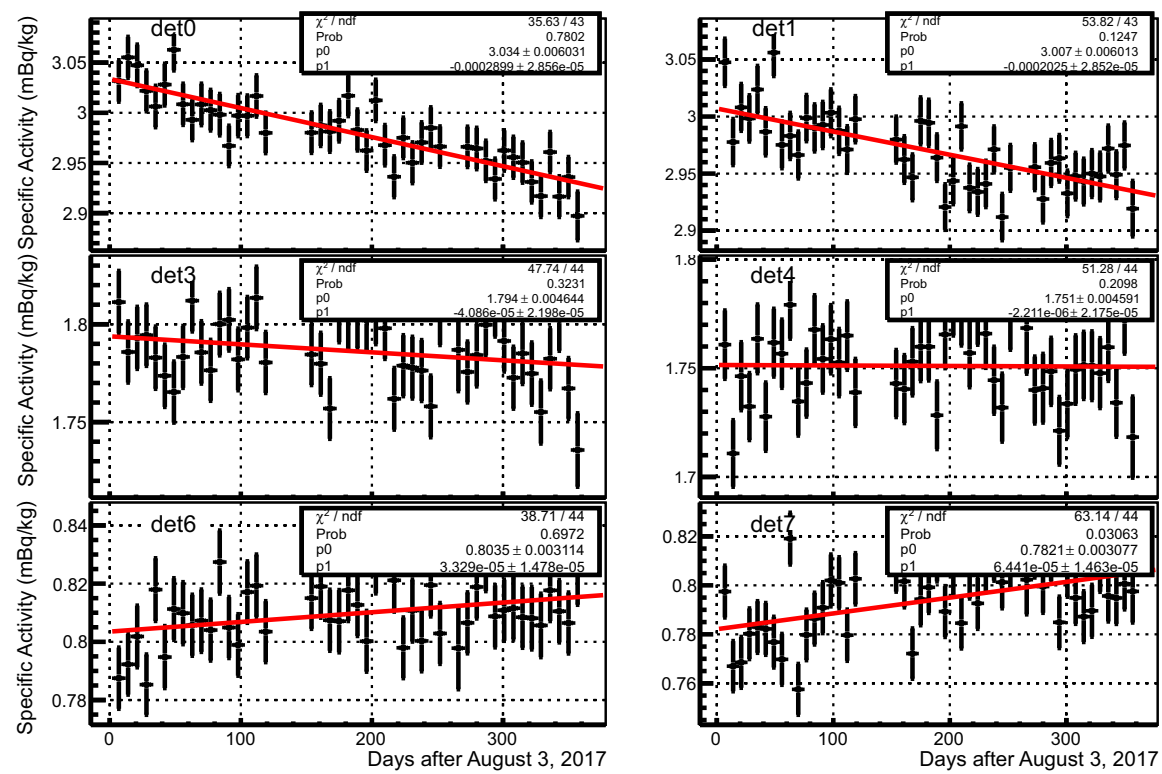

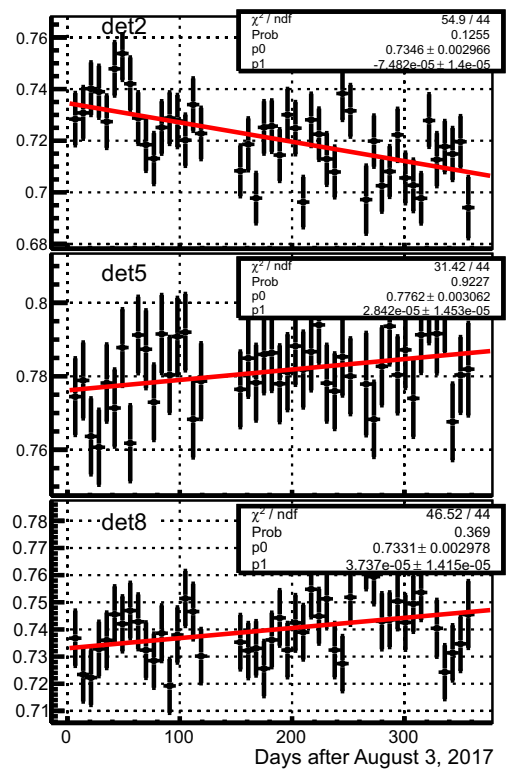

Fig. 34 Evolution of the specific activity of alpha events attributed mainly to ${ }^{210}$ Po decay in the crystal bulk. Alpha events are selected by PSA (see Sect. 3.1 and Fig. 11). Rates are averaged per week and linearly fitted. It can be observed that modules arriving first at Canfranc (D0, D1,
D2 and D3) show a decaying alpha rate because equilibrium in the chain was reached and ${ }^{210} \mathrm{~Pb}$ decay time constant rules the ${ }^{210} \mathrm{Po}$ decay; for modules arriving later at Canfranc, rate of alpha events is either constant or slightly increasing because ${ }^{210} \mathrm{Po}$ activity is still being built radon content in laboratory air (inside ANAIS hut); relative humidity in several places; HV supply for every PMT; electronics voltage supply; etc. All the data are saved every few minutes and alarms have been set on the most relevant parameters sending an alarm message through Telegram. Very few incidents have set on the ANAIS-112 alarms along the first year of data taking, only the radon-free nitrogen gas flux entering into the shielding which has been reduced down to zero three times in the data taking because of exhausting the liquid nitrogen dewar, as already explained in Sect. 2. Figure 36 shows the evolution of the radon content in the air of the ANAIS hut (but outside the shielding) along the first year 


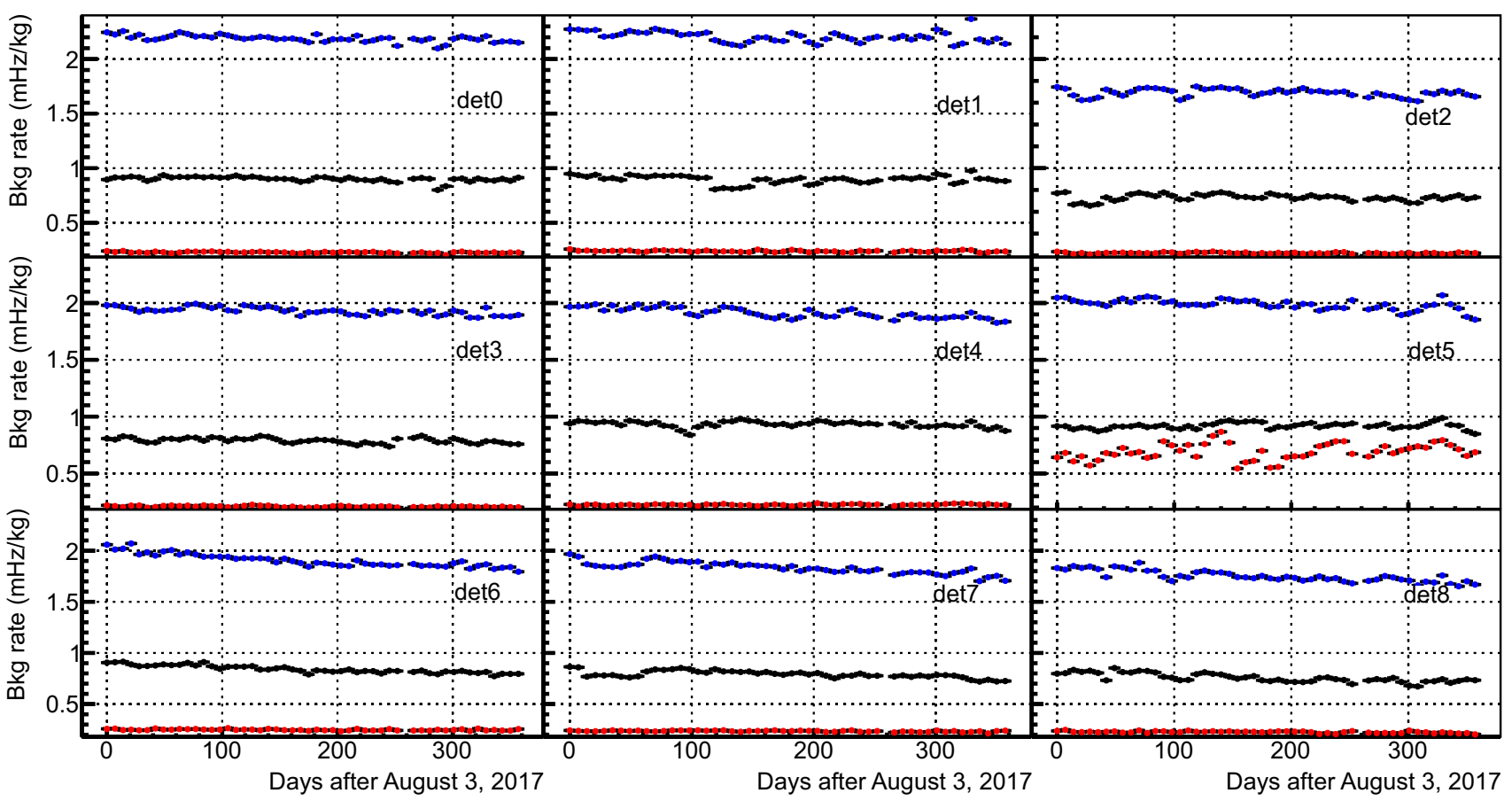

Fig. 35 Evolution of the rate of events attributed to interactions of background gammas in the crystal bulk in three different energy windows (in black around the $609.3 \mathrm{keV}$ line (from 559 to $659 \mathrm{keV}$ ), in red around the $1460.8 \mathrm{keV}$ line (from 1410 to $1510 \mathrm{keV}$ ) and in blue in the region from 300 to $450 \mathrm{keV}$ ). Rates are averaged per week. Colors referenced are available in the online version of the paper of data taking. It has to be remarked that radon content inside the shielding is expected to be about two orders of magnitude lower than that thanks to the radon-free nitrogen gas overpressure. We show in Fig. 37 the time behaviour of the nitrogen gas flux entering into the ANAIS-112 shielding and in Fig. 38 the relative humidity in different positions (inside the ANAIS hut and at the electronics air conditioned space). There is a clear correlation between relative humidity and the radon content in the lab air presented in Fig. 36. For both a fit of the data to a function $f(t)=p_{0}+p_{1} \cdot \cos \left(2 \pi p_{2}\left(t+p_{3}\right)\right)$ is performed, showing clear differences in the best fit for the period of the oscillation ( $400 \pm 56$ days vs $630 \pm 50$ days). Further information on this correlation will be obtained by accumulating more years of data.

Figures 39 and 40 show the evolution in time along the first year of ANAIS-112 data taking of the temperature inside the ANAIS-112 shielding (Tint), the temperature at the electronics air-conditioned space (Telcabin), and the temperatures at VME and NIM racks placed inside that conditioned space (Tvme, Tnim1, Tnim2). The temperature at the detectors position, Tint, follows the external conditions at the LSC hall B. It varies from 18.09 to $19.85^{\circ} \mathrm{C}$, having a mean value of $19.24^{\circ} \mathrm{C}$ and standard deviation of $0.48^{\circ} \mathrm{C}$. The temperature of the preamplifiers follows exactly the same trend, while on the other hand, temperature at the VME and NIM electronics racks is fully decoupled from hall B temperature. The latter

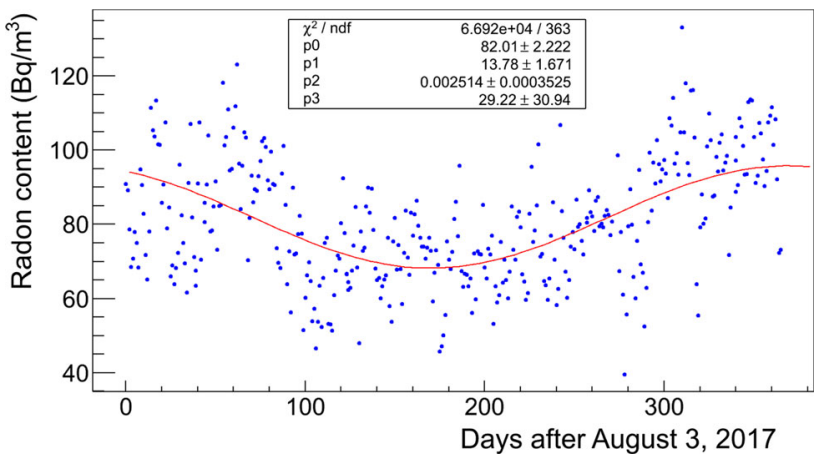

Fig. 36 Evolution of the radon content in the LSC hall B air in the ANAIS hut outside the shielding. Rates are calculated per day and fitted to a cosinoidal dependence

has been quite stable along the first year of data taking but for sudden changes (see Fig. 40), which are due to adjustments in the configuration of the temperature control and accidental shift of the temperature sensors while maintenance works.

\section{Summary}

ANAIS-112 experiment has been taking data at the Canfranc Undeground Laboratory for more than one year, and the experiment goal is to accumulate five years of data in sta- 


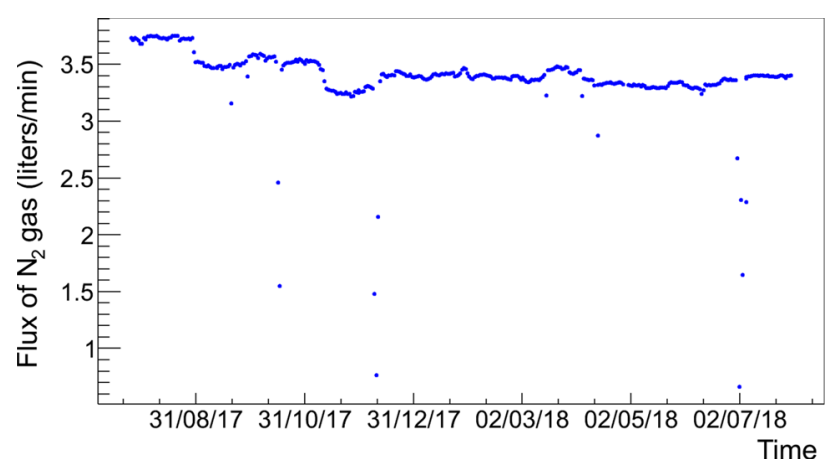

Fig. 37 Evolution of the nitrogen gas flux entering into the ANAIS112 shielding to prevent radon intrusion, averaged per day

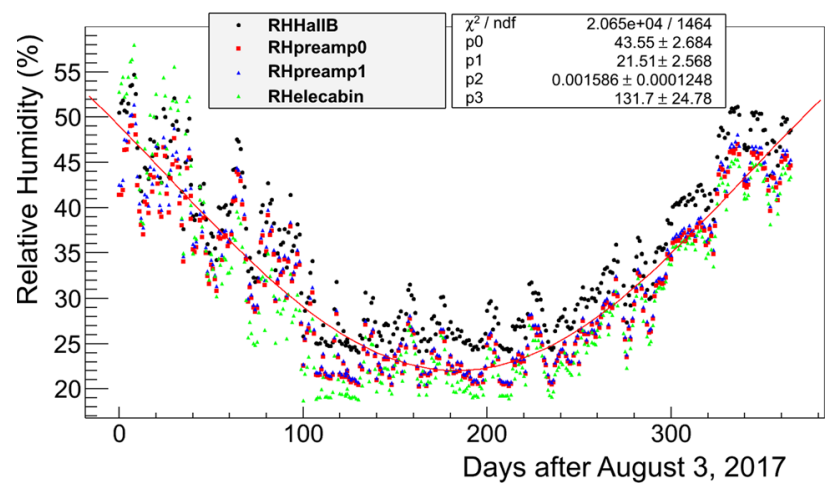

Fig. 38 Evolution of the Relative Humidity inside the ANAIS hut (RHHallB), at the preamplifiers positions (RHpreamp0, RHpreamp1) and at the ANAIS-112 electronics air-conditioned room (RHelecabin). All of them have been averaged per day and fitted to a cosinoidal dependence. Colors referenced are available in the online version of the paper

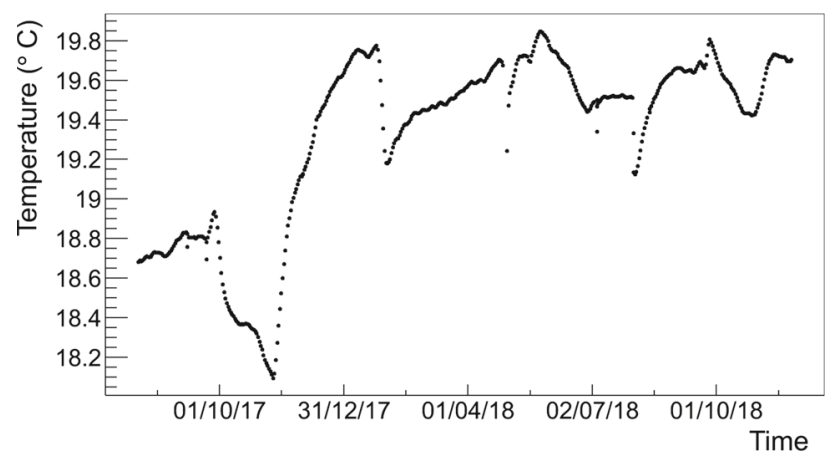

Fig. 39 Evolution of the temperature inside the ANAIS-112 shielding (Tint), averaged per day. Tint follows the external conditions at LSC hall B. In the first year of operation, it varies from 18.09 to $19.85^{\circ} \mathrm{C}$, having a mean value of $19.24^{\circ} \mathrm{C}$ and standard deviation of $0.48^{\circ} \mathrm{C}$. LSC temperature regulation is based on sensors placed in hall $\mathrm{A}$, and then, temperature at hall $\mathrm{B}$ is affected, for instance by the operation of ArDM experiment at hall $\mathrm{A}$, which introduces an additional heat source and forces the system to cool down, which is observed in the figure in November 2017. Efforts of the LSC staff to improve the hall B temperature control are ongoing, and along 2018 variations in temperature at hall B have been much smaller. No correlation among Tint and ANAIS-112 trigger rate has been observed along the first year of operation

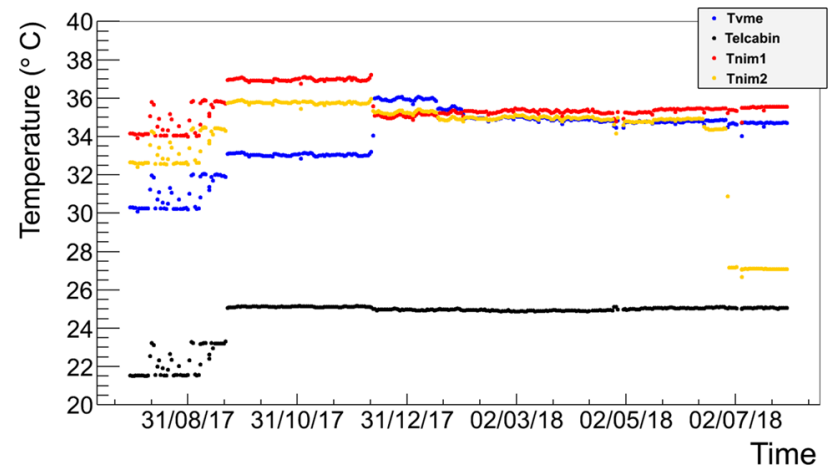

Fig. 40 Evolution of the temperatures inside the ANAIS-112 electronics air-conditioned room (Telcabin), and at different positions of the electronics racks (Tvme, Tnim1, Tnim2). All of them have been averaged per day. Colors referenced are available in the online version of the paper

ble conditions in order to test the DAMA/LIBRA modulation signal in a model independent way. An excellent duty cycle has been achieved, accumulating 341.72 days of data with the nine $\mathrm{NaI}(\mathrm{Tl})$ modules (12.5 kg each). ANAIS-112 modules have shown a very high light collection per unit of energy deposited, about 15 phe/ $\mathrm{keV}$ in 8 out of 9 modules. In this article, the performance of the ANAIS-112 experiment along the first year of data taking has been reviewed, as well as the analysis techniques developed for low energy calibration, in particular, selection of events corresponding to interactions attributable to dark matter, and the corresponding efficiencies have been thoroughly described and estimated. Background events populations in the ROI, from ${ }^{40} \mathrm{~K}$ and ${ }^{22} \mathrm{Na}$ decays in the crystal bulk, are well tagged and used to control any modulation in the operation of the detectors that could mimic the signal searched for. Environmental parameters (muon flux, radon content in the laboratory air, temperature at the detectors positions and electronics, etc.) are being continuously monitored. ANAIS-112 will be able to test DAMA/LIBRA signal at $3 \sigma$ level in five years of data taking in the achieved experimental conditions, having a large discovery potential if dark matter particles are responsible for such signal. Estimates of the ANAIS-112 sensitivity prospects have been updated [40] with the background level corresponding to the unblinded data and the efficiencies reported in this work.

Acknowledgements This work has been financially supported by the Spanish Ministerio de Economía y Competitividad and the European Regional Development Fund (MINECO-FEDER) under grants No. FPA2014-55986-P and FPA2017-83133-P, the Consolider-Ingenio 2010 Programme under grants MultiDark CSD2009-00064 and CPAN CSD2007-00042 and the Gobierno de Aragón and the European Social Fund (Group in Nuclear and Astroparticle Physics and I. Coarasa predoctoral grant). We thank the support of the Spanish Red Consolider MultiDark FPA2017-90566-REDC. We acknowledge the technical support from LSC and GIFNA staff. We also would like to acknowledge the use of Servicio General de Apoyo a la Investigación-SAI, Univer- 
sidad de Zaragoza. Professor J.A. Villar passed away in August, 2017. Deeply in sorrow, we all thank his dedicated work and kindness.

Data Availability Statement This manuscript has no associated data or the data will not be deposited. [Author's comment: ANAIS-112 data will be released after the intended five-year operation, and corresponding exploitation and publication of results.]

Open Access This article is distributed under the terms of the Creative Commons Attribution 4.0 International License (http://creativecomm ons.org/licenses/by/4.0/), which permits unrestricted use, distribution, and reproduction in any medium, provided you give appropriate credit to the original author(s) and the source, provide a link to the Creative Commons license, and indicate if changes were made.

Funded by SCOAP ${ }^{3}$.

\section{References}

1. G. Bertone, D. Hooper, Rev. Mod. Phys. 90(4), 045002 (2018). https://doi.org/10.1103/RevModPhys.90.045002

2. L. Baudis, J. Phys. G43(4), 044001 (2016). https://doi.org/10. 1088/0954-3899/43/4/044001

3. T. Marrodán Undagoitia, L. Rauch, J. Phys. G43(1), 013001 (2016). https://doi.org/10.1088/0954-3899/43/1/013001

4. J.M. Gaskins, Contemp. Phys. 57(4), 496 (2016). https://doi.org/ 10.1080/00107514.2016.1175160

5. E.A. Bagnaschi et al., Eur. Phys. J. C 75, 500 (2015). https://doi. org/10.1140/epjc/s10052-015-3718-9

6. R. Bernabei et al., Eur. Phys. J. C 56, 333 (2008). https://doi.org/ 10.1140/epjc/s10052-008-0662-y

7. R. Bernabei et al., Eur. Phys. J. C 73, 2648 (2013). https://doi.org/ 10.1140/epjc/s10052-013-2648-7

8. R. Bernabei et al., (2018). arXiv:1805.10486

9. C. Savage, K. Freese, P. Gondolo, D. Spolyar, JCAP 0909, 036 (2009). https://doi.org/10.1088/1475-7516/2009/09/036

10. E. Aprile et al., Science 349(6250), 851 (2015). https://doi.org/10. 1126/science.aab2069

11. J. Herrero-Garcia, JCAP 1509(09), 012 (2015). https://doi.org/10. 1088/1475-7516/2015/09/012

12. S. Baum, K. Freese, C. Kelso, Phys. Lett. B 789, 262 (2019). https:// doi.org/10.1016/j.physletb.2018.12.036

13. S. Kang, S. Scopel, G. Tomar, J.H. Yoon, JCAP 1807(07), 016 (2018). https://doi.org/10.1088/1475-7516/2018/07/016

14. J. Herrero-Garcia, A. Scaffidi, M. White, A.G. Williams, Phys. Rev. D 98(12), 123007 (2018). https://doi.org/10.1103/PhysRevD. 98.123007

15. I. Coarasa et al., (2017). arXiv:1704.06861

16. K.W. Kim et al., (2018). arXiv:1806.06499
17. G. Adhikari et al., Eur. Phys. J. C 78(2), 107 (2018). https://doi. org/10.1140/epjc/s10052-018-5590-X

18. C. Tomei, Nucl. Instrum. Methods A845, 418 (2017). https://doi. org/10.1016/j.nima.2016.06.007

19. A. Gütlein, Nucl. Instrum. Methods A845, 359 (2017). https://doi. org/10.1016/j.nima.2016.06.005

20. J. Amaré et al., Eur. Phys. J. C 76(8), 429 (2016). https://doi.org/ 10.1140/epjc/s10052-016-4279-2

21. J. Amaré et al., Eur. Phys. J. C. arXiv:1812.01377

22. M. Oliván, Ph.D. thesis, Universidad de Zaragoza (2016). arXiv: $1601.07312 \mathrm{v} 1$

23. M. Agostini et al., (2018). arXiv:1808.04207

24. M. Agostini et al., Astropart. Phys. 84, 29 (2016). https://doi.org/ 10.1016/j.astropartphys.2016.08.002

25. J. Cherwinka et al., Phys. Rev. D 93(4), 042001 (2016). https://doi. org/10.1103/PhysRevD.93.042001

26. M. Ambrosio et al., Phys. Rev. D 67, 042002 (2003). https://doi. org/10.1103/PhysRevD.67.042002

27. R. Bernabei et al., Eur. Phys. J. C 72, 2064 (2012). https://doi.org/ 10.1140/epjc/s10052-012-2064-4

28. J. Amaré, Opt. Mater. 36, 1408 (2014). https://doi.org/10.1016/j. optmat.2014.03.042

29. C. Cuesta, Opt. Mater. 36, 316 (2013). https://doi.org/10.1016/j. optmat.2013.09.015

30. I. Bandac, J. Phys. Conf. Ser. 934(1), 012019 (2017). https://doi. org/10.1088/1742-6596/934/1/012019

31. M.A. Oliván et al., Astropart. Phys. 93, 86 (2017). https://doi.org/ 10.1016/j.astropartphys.2017.06.005

32. R. Brun, F. Rademakers, Nucl. Instrum. Methods A 389, 250 (1997). http://root.cern.ch. Accessed 7 Mar 2019

33. S.Y.F. Chu, L.P. Ekstrom, R.B. Firestone, The lund/lbnl nuclear data search. http://nucleardata.nuclear.lu.se/toi/xray.asp?act=list\& el=Ag. Accessed 7 Mar 2019

34. S.Y.F. Chu, L.P. Ekstrom, R.B. Firestone, The lund/lbnl nuclear data search. http://nucleardata.nuclear.lu.se/toi/xray.asp?act=list\& $\mathrm{el}=\mathrm{Br}$. Accessed 7 Mar 2019

35. R. Bernabei, Nucl. Instrum. Methods Phys. Res. A 592, 297 (2008). https://doi.org/10.1016/j.nima.2008.04.082

36. M. Tanabashi et al., Phys. Rev. D 98, 030001 (2018). https://doi. org/10.1103/PhysRevD.98.030001

37. D.Y. Akimov, A.I. Bolozdynya, Y.V. Efremenko, V.A. Kaplin, A.V. Khromov, Y.A. Melikyan, V.V. Sosnovtsev, Nucl. Instrum. Methods A794, 1 (2015). https://doi.org/10.1016/j.nima.2015.04.066

38. S. Li et al., JINST 11(02), T02005 (2016). https://doi.org/10.1088/ 1748-0221/11/02/T02005

39. C. Cuesta et al., Eur. Phys. J. C 74(11), 3150 (2014). https://doi. org/10.1140/epjc/s10052-014-3150-6

40. I. Coarasa et al., Eur. Phys. J. C. https://doi.org/10.1140/epjc/ s10052-019-6733-4. arXiv: 1812.02000 